\title{
WAVEGUIDES COUPLED THROUGH A SEMITRANSPARENT BARRIER: A BIRMAN-SCHWINGER ANALYSIS
}

P. EXNER

Department of Theoretical Physics, NPI

Academy of Sciences, 25068 Řež near Prague and Doppler Institute, Czech Technical University

Břehová 7, 11519 Prague, Czechia

exner@ujf.cas.cz

D. KREJČIŘÍK

Department of Theoretical Physics Institute, NPI AS, Faculty of Mathematics and Physics, Charles University

V Holešovičkách 2, 18000 Prague, Czechia, and Faculté des Sciences et Technologies Université de Toulon et du Var BP 132, 83957 La Garde Cedex, France krejcirik@ujf.cas.cz

The paper is devoted to a model of a mesoscopic system consisting of a pair of parallel planar waveguides separated by an infinitely thin semitransparent boundary modeled by a transverse $\delta$ interaction. We develop the Birman-Schwinger theory for the corresponding generalized Schrödinger operator. The spectral properties become nontrivial if the barrier coupling is not invariant with respect to longitudinal translations, in particular, there are bound states if the barrier is locally more transparent in the mean and the coupling parameter reaches the same asymptotic value in both directions along the guide axis. We derive the weak-coupling expansion of the ground-state eigenvalue for the cases when the perturbation is small in the supremum and the $\boldsymbol{L}^{1}$-norms. The last named result applies to the situation when the support of the leaky part shrinks: the obtained asymptotics differs from that of a double guide divided by a pierced Dirichlet barrier. We also derive an upper bound on the number of bound states . 


\section{Contents}

1 Introduction 2

1.1 Motivation . . . . . . . . . . . . . . . 2

1.2 Description of the model and contents of the paper . . . . . . 4

2 Singularly supported interactions on

$\begin{array}{ll}\text { a subset of } \mathbb{R}^{d} & 5\end{array}$

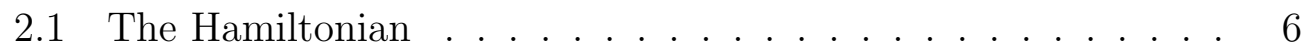

2.2 Auxiliary results $\ldots \ldots \ldots$. . . . . . . . . . . 6

2.3 The resolvent $\ldots \ldots \ldots \ldots$

3 BS analysis for the double waveguide 13

3.1 The basic lemma . . . . . . . . . . . . . . . . . . . . . . 13

3.2 The resolvent comparison formula . . . . . . . . . . . . . . . 16

\begin{tabular}{|lr}
4 Weak coupling & 19
\end{tabular}

4.1 Preliminaries . . . . . . . . . . . . . . . . . . . . . . . . 19

4.2 Existence of the ground state . . . . . . . . . . . . . . . . 22

4.3 Weak-coupling expansion . . . . . . . . . . . . . . . . . . . . . 24

5 Narrow-window coupling $\quad 25$

5.1 Motivation: squeezing the "leaky" part . . . . . . . . . . . . 25

5.2 Modified lemmas . . . . . . . . . . . . . . . 26

5.3 The results for the scaled case . . . . . . . . . . . . . . . . . . 28

6 A bound on the number of eigenvalues 30

6.1 A general SKN-type bound . . . . . . . . . . . . . . . . . 30

6.2 A "rectangular well" example . . . . . . . . . . . . . . 32

\section{Introduction}

\subsection{Motivation}

The recent progress of solid-state physics opened way to testing of quantum mechanics in hitherto unusual situations. Many of the "mesoscopic" semiconductor systems can be regarded as electron waveguides in which wave 


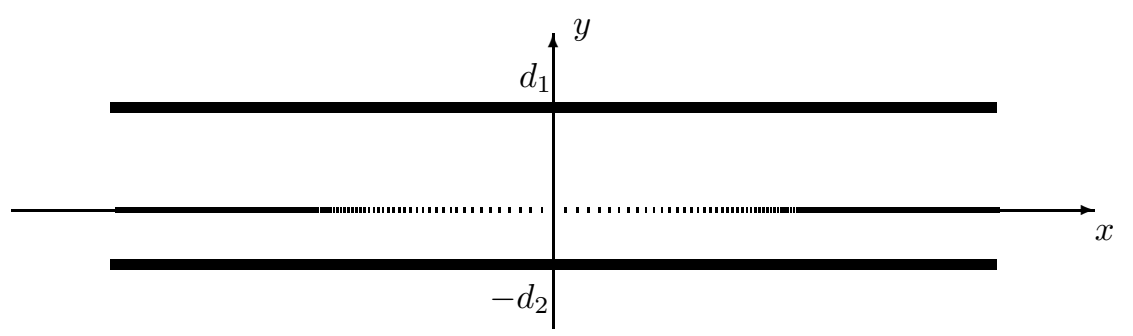

Figure 1: Double waveguide with a $\delta$ barrier

properties of the particles play an essential role - we refer to [6, 9] for discussion of the model assumptions involved and a bibliography.

An interesting class of such systems is represented by a pair of parallel planar guides with a lateral coupling, which is realized either by a "window" in a Dirichlet barrier separating the ducts [4, 9] or by a local variation of the coupling parameter in a leaky, i.e., semitransparent barrier [8]. In the latter case (sketched in Figure 1) the Hamiltonian is formally given by the relation (1.1) below, with the barrier supported by the $x$-axis. The function $\alpha$ describes the coupling parameter and the outer boundary of the double strip $\Omega:=\mathbb{R} \times\left(-d_{2}, d_{1}\right)$ is supposed to be hard, i.e., we impose Dirichlet boundary conditions there.

Depending on the choice of $\alpha$, such a model describe a variety of different dynamical situations. It is illustrative to consider the case related to the example of a pierced-hard-wall discussed in Ref. [9]; the comparison being based on the fact that the $\delta$ interaction with a large coupling constant approximates the Dirichlet barrier. The example of a step-function-shaped $\alpha$ analyzed in Refs. [8] exhibits indeed for large $\alpha$ close similarities in the numerically calculated shapes of the eigenfunctions, etc. At the same time, asymptotic properties of the eigenvalues may be rather different in the two cases if we exclude here the possibility $\alpha=\infty$ which expresses formally the Dirichlet boundary condition - $c f$. Remarks 5.7.

Systems with a $\delta$ potential barrier of the type (1.1) are mathematically more accessible, since two operators with different functions $\alpha$ have the same form domain. This observation will make it possible to construct a BirmanSchwinger-type theory in this case writing down an explicit expression for the difference between the resolvent of the Hamiltonian (1.1) and that of a suitable comparison operator. The main consequence of this formula which we derive in this paper is the weak coupling expansion in the situation when 
$\alpha$ forms a "potential well", i.e., when the barrier is locally more transparent (at least in the mean) and the coupling parameter $\alpha(x)$ reaches the same asymptotic value in both directions along the guide axis.

\subsection{Description of the model and contents of the paper}

As we have said, the configuration space of our system is a straight planar strip $\Omega:=\mathbb{R} \times \mathcal{O}$ with $\mathcal{O}:=\mathcal{O}_{2} \cup \mathcal{O}_{1}:=\left(-d_{2}, 0\right) \cup\left(0, d_{1}\right)$ in which the free motion is restricted by the outer hard walls and a $\delta$ potential barrier at $y=0$. Its coupling strength $\alpha \in \mathbb{R}$ varies longitudinally, $\alpha=\alpha(x)$, so the particle Hamiltonian can be formally written as

$$
H_{\alpha}=-\Delta_{D}^{\Omega}+\alpha(x) \delta(y) .
$$

There are several equivalent ways to give the right-hand-side of (1.1) a rigorous meaning. Following [2, Chap. I.3] this can be done by imposing the standard boundary conditions [8]. In this paper, however, we use instead a quadratic-form definition which is much more general. Such generalized Schrödinger operators in $\mathbb{R}^{d}$ with a measure-induced interaction were studied in [3]. In the next section we shall adapt this theory for the case when the free Hamiltonian is the Dirichlet Laplacian relative to a subset $\Omega \subset \mathbb{R}^{d}$. To make the paper self-contained, we outline the construction from Sec. 2 of the mentioned paper with emphasis on the modifications required by the presence of the Dirichlet boundary. This concerns mostly Lemma 2.2 whose proof in [3] relies on the explicit form of the free Green's function.

In Section 3 we shall use this results to formulate the Birman-Schwinger theory for the operator (1.1). The basic idea is again adopted from [3], however, it suits to our purpose to express the resolvent difference with respect to a comparison operator which also has a nonzero $\alpha$, and to write it in a symmetric form.

The obtained resolvent formula is then employed to investigate the discrete spectrum of our Hamiltonian which exists if the $\delta$ barrier produces a local attractive interaction. Sections 4 and 5 are devoted the weak-coupling analysis of our model in two situations: in the first case the interaction is tuned by means of a coupling constant, in the second one we use instead a scaling transformation with respect to the longitudinal variable. In both situations we derive an asymptotic expansion for the ground state eigenvalue. In the example which involves the scaling the "potential well" given by the 
shape of the function $\alpha$ may be deep, the weak coupling being achieved by its narrowness. This makes it possible to compare the asymptotics with the mentioned Dirichlet case, where the gap is proportional to the fourth power of the window width. In contrast, for any "soft" barrier the width appears in the leading term of the asymptotics with the square only.

In the final section we use the Birman-Schwinger technique to derive an upper bound on the dimension of the discrete s pectrum. A comparison with the "square well" example of [8] shows that the bound is good for weak coupling but its semiclassical behavior is not correct as it is the case for the usual Schrödinger operators [14].

\section{Singularly supported interactions on a subset of $\mathbb{R}^{d}$}

Let $\Omega$ be an open subset of $\mathbb{R}^{d}$. Consider a positive Radon measure $m$ on $\Omega$, i.e. the abstraction of Lebesgue's outer measure for general topological spaces [16, Def. 2.3.9], and a Borel measurable function $\alpha: \mathbb{R}^{d} \rightarrow \mathbb{R}$ such that

$$
\int_{\Omega}|\psi(x)|^{2}\left(1+\alpha(x)^{2}\right) d m(x) \leq a \int_{\Omega}|\nabla \psi(x)|^{2} d x+b \int_{\Omega}|\psi(x)|^{2} d x
$$

holds for all $\psi \in \boldsymbol{C}_{0}^{\infty}(\Omega)$ and some positive $a<1$ and $b$. As indicated in the introduction, we are interested mainly in situation when $m$ is a $\delta$-measure supported by a planar curve, but the argument presented below does not need such restrictions on the measure or space dimension; it includes also the regular potential case, $d m(x)=|V(x)| d x$.

By definition, $\boldsymbol{C}_{0}^{\infty}(\Omega)$ is dense in the local Sobolev space $\boldsymbol{W}_{0}^{2,1}(\Omega)(c f$. [17, Sec. XIII.14]), so there is a unique bounded linear operator

$$
I_{m}: \boldsymbol{W}_{0}^{2,1}(\Omega) \rightarrow \boldsymbol{L}^{2}(m):=\boldsymbol{L}^{2}(\Omega, d m)
$$

such that $I_{m} \psi=\psi$ is valid for any $\psi \in \boldsymbol{C}_{0}^{\infty}(\Omega)$. The last relation means in fact $\left(I_{m} \psi\right)(x)=\psi(x)$ for $x \in \operatorname{supp} m$; with an abuse of notation we shall employ the symbol $\psi$ for $(i)$ a continuous function $\psi$, (ii) the corresponding

$\boldsymbol{L}^{2}(\Omega)$ equivalence class, and finally (iii) for the corresponding $\boldsymbol{L}^{2}(\Omega, d m)$ equivalence class. By density, the inequality (2.1) holds for all $\psi \in \boldsymbol{W}_{0}^{2,1}(\Omega)$ provided $\psi$ is replaced by $I_{m} \psi$ on the left-hand-side. 


\subsection{The Hamiltonian}

We introduce the following quadratic form

$$
\mathcal{E}_{\alpha m}(\psi, \varphi):=\int_{\Omega} \overline{\nabla \psi(x)} \cdot \nabla \varphi(x) d x+\int_{\Omega} \alpha(x)\left(I_{m} \bar{\psi}\right)(x)\left(I_{m} \varphi\right)(x) d m(x)
$$

with the domain $D\left(\mathcal{E}_{\alpha m}\right)=\boldsymbol{W}_{0}^{2,1}(\Omega)$. It is well known [17, Sec. XIII.14] that the free form $\mathcal{E}_{0}$ is positive and closed on $\boldsymbol{L}^{2}(\Omega)$; it gives rise to the Dirichlet Laplacian $-\Delta_{D}^{\Omega}$. By the KLMN theorem [17, Thm. X.17] and the extended version of inequality (2.1), $\mathcal{E}_{\alpha m}$ is lower semibounded and closed on $\boldsymbol{L}^{2}(\Omega)$, and $C_{0}^{\infty}(\Omega)$ is a core for it. Hence by the second representation theorem, there is a unique self-adjoint operator $H_{\alpha m}$ associated with $\mathcal{E}_{\alpha m}$; it will the object of our interest in the following.

The basic assumption (2.1) is satisfied, in particular, for measures $m$ belonging to the generalized Kato class. By 20 the inequality (2.1) holds for such $m$ and $\psi \in \mathcal{S}\left(\mathbb{R}^{d}\right)$, and the same is, a fortiori, true for $\psi \in \boldsymbol{C}_{0}^{\infty}(\Omega)$ corresponding to an open $\Omega \subset \mathbb{R}^{d}$. If $d=2$ the Kato condition reads

$$
\lim _{\varepsilon \rightarrow 0+} \sup _{x \in \Omega} \int_{B(x, \varepsilon) \cap \Omega}|\ln | x-y|| d m(y)=0,
$$

where $B(x, \varepsilon)$ is the ball of radius $\varepsilon$ and center $x$. It is straightforward to check that the condition is satisfied for the $\delta$ measure of our example; alternatively one can employ Thm. 4.1 of [3].

\section{$2.2 \quad$ Auxiliary results}

We will need two lemmas. The first one is abstract and we adopt it from [3]:

Lemma 2.1 Let $\mathcal{E}$ be a lower semibounded densely defined closed quadratic form on a complex Hilbert space $\mathcal{H}$ with the inner product $(\cdot, \cdot)$, and let $H$ be the unique self-adjoint operator on $\mathcal{H}$ associated with $\mathcal{E}$. Finally, let $R: \mathcal{H} \rightarrow D(\mathcal{E})$ be an arbitrary map and $z \in \mathbb{C}$. Then the following statements are equivalent.

(i) $z \in \rho(H)$ and $(H-z)^{-1}=R$.

(ii) $\forall \psi \in \mathcal{H}, \varphi \in D(\mathcal{E}): \quad \mathcal{E}(R \psi, \varphi)=(z R \psi+\psi, \varphi)$. 
Let now $\mathbb{C}_{\Omega, 0}^{+}$be the set $\left\{k: \operatorname{Im} k>0\right.$ or $\left.k^{2} \in\left[0, \inf \sigma\left(-\Delta_{D}^{\Omega}\right)\right)\right\} \subset \mathbb{C}$. Given $k \in \mathbb{C}_{\Omega, 0}^{+}$we denote by $G_{0}(\cdot, \cdot ; k)$ the free resolvent kernel for $z=k^{2}$ corresponding to the Dirichlet Laplacian $-\Delta_{D}^{\Omega}$. The main difference with respect to [3] is that for $\Omega \neq \mathbb{R}^{d}$ the kernel depends on both arguments, not just on their difference.

Let $\mu, \nu$ be positive Radon measures without a discrete component, i.e. $\mu(\{a\})=\nu(\{a\})=0$ for any $a \in \Omega$. We denote by $R_{\mu, \nu}^{k}$ the integral operator from $\boldsymbol{L}^{2}(\mu):=\boldsymbol{L}^{2}(\Omega, d \mu)$ to $\boldsymbol{L}^{2}(\nu)$ with the kernel $G_{0}(\cdot, \cdot ; k)$. In particular, we have

$$
\left(R_{\mu, \nu}^{k} \psi\right)(x)=\int_{\Omega} G_{0}(x, y ; k) \psi(y) d \mu(y)
$$

for all $\psi \in D\left(R_{\mu, \nu}^{k}\right) \subset \boldsymbol{L}^{2}(\mu)$. Since we agreed to the mentioned abuse of notation, there is no $\nu$ in the definition; we compute the right-hand-side and interpret it as values of a function in $\boldsymbol{L}^{2}(\nu)$. In the following $(\cdot, \cdot)$ will denote the inner product on $\boldsymbol{L}^{2}(\Omega)$.

Lemma 2.2 Let $k \in \mathbb{C}_{\Omega, 0}^{+}$and $\psi \in \boldsymbol{L}^{2}(m)$. Then $R_{m, d x}^{k} \psi \in \boldsymbol{W}_{0}^{2,1}(\Omega)$ and

$$
\forall \varphi \in \boldsymbol{W}_{0}^{2,1}(\Omega): \quad \mathcal{E}_{0}\left(R_{m, d x}^{k} \psi, \varphi\right)-\left(k^{2} R_{m, d x}^{k} \psi, \varphi\right)=\int_{\Omega} \bar{\psi}(y)\left(I_{m} \varphi\right)(y) d m(y)
$$

In particular, $R_{m, d x}^{k}$ is injective.

PROOF: If we prove the above relation, the injectivity will follow by density of Ran $I_{m}$ in $\boldsymbol{L}^{2}(m)$. Assume first $k^{2}<0$, i.e., $k$ is purely imaginary. Then

$$
\langle\psi, \varphi\rangle_{k}:=\mathcal{E}_{0}(\psi, \varphi)-k^{2}(\psi, \varphi)
$$

defines an inner product on $\boldsymbol{W}_{0}^{2,1}(\Omega)$ and the corresponding norm is equivalent to the usual Sobolev norm (with $k^{2}=-1$ ). Take a fixed $\psi \in \boldsymbol{L}^{2}(m)$. Using Schwarz inequality and the fact that $I_{m}$ is bounded we infer

$$
\begin{aligned}
\left|\int_{\Omega} \bar{\psi}(y)\left(I_{m} \varphi\right)(y) d m(y)\right|^{2} & \leq \int_{\Omega}|\psi(y)|^{2} d m(y) \int_{\Omega}\left|\left(I_{m} \varphi\right)(y)\right|^{2} d m(y) \\
& \leq c\langle\varphi, \varphi\rangle_{k}
\end{aligned}
$$

for any $\varphi \in \boldsymbol{W}_{0}^{2,1}(\Omega)$ and some constant $c$ depending on $\psi$. Hence the linear functional

$$
\varphi \mapsto \int_{\Omega} \bar{\psi}(y)\left(I_{m} \varphi\right)(y) d m(y)
$$


on the Hilbert space $\left(\boldsymbol{W}_{0}^{2,1}(\Omega),\langle\cdot, \cdot\rangle_{k}\right)$ is bounded, and by Riesz's lemma, there is a unique $\psi_{m}^{k} \in \boldsymbol{W}_{0}^{2,1}(\Omega)$ such that

$$
\forall \varphi \in \boldsymbol{W}_{0}^{2,1}(\Omega): \quad\left\langle\psi_{m}^{k}, \varphi\right\rangle_{k}=\int_{\Omega} \bar{\psi}(y)\left(I_{m} \varphi\right)(y) d m(y) .
$$

Consequently, it is sufficient to show that

$$
\forall \psi \in \boldsymbol{L}^{2}(\Omega): \quad\left(R_{m, d x}^{k} \psi\right)(x) \equiv \int_{\Omega} G_{0}(x, y ; k) \psi(y) d m(y)=\psi_{m}^{k}(x)
$$

a.e. with respect to the Lebesgue measure $d x$.

If $\Omega \neq \mathbb{R}^{d}$ we have in general no explicit expression for the Green's function $G_{0}(x, y ; k)$ with a given $k^{2}<0$. We know, however, that it is positive for all $x, y \in \Omega, x \neq y$ [17, App. 1 to Sec. XIII.12], and moreover, that the kernel is $d x$-integrable if the other variable is fixed (in fact, exponentially decaying for a non-compact $\Omega$ ) and

$$
\forall y \in \Omega, \varphi \in \boldsymbol{W}_{0}^{2,1}(\Omega): \quad \int_{\Omega} G_{0}(x, y ; k)\left(-\Delta_{D}^{\Omega}-k^{2}\right) \varphi(x) d x=\varphi(y) .
$$

Functions $\varphi:=\left(-\Delta_{D}^{\Omega}-k^{2}\right)^{-1} \eta$ with $\eta \in \boldsymbol{C}_{0}^{\infty}(\Omega)$ are bounded $\boldsymbol{C}^{\infty}$ and have the same decay as the Green's function for a non-compact $\Omega$.

We shall prove the desired relation in several steps. Suppose first that $\psi \in \boldsymbol{L}^{1}(m) \cap \boldsymbol{L}^{2}(m)$. For $\varphi$ of the described class we may employ then the Fubini theorem obtaining

$$
\begin{aligned}
\int_{\Omega} \overline{\left(\int_{\Omega} G_{0}(x, y ; k) \psi(y) d m(y)\right)}\left(-\Delta_{D}^{\Omega}-k^{2}\right) \varphi(x) d x \\
\quad=\int_{\Omega} \bar{\psi}(y)\left(\int_{\Omega} G_{0}(y, x ; k)\left(-\Delta_{D}^{\Omega}-k^{2}\right) \varphi(x) d x\right) d m(y) \\
=\int_{\Omega} \bar{\psi}(y) \varphi(y) d m(y)
\end{aligned}
$$

We have used here also the fact that $G_{0}$ is real-valued for $k^{2}<0$. By (2.6) and the second representation theorem, we have

$$
\int_{\Omega} \overline{\psi_{m}^{k}}\left(-\Delta_{D}^{\Omega}-k^{2}\right) \varphi(x) d x=\left\langle\psi_{m}^{k}, \varphi\right\rangle_{k}=\int_{\Omega} \bar{\psi}(y) \varphi(y) d m(y)
$$

for all $\varphi \in\left(-\Delta_{D}^{\Omega}-k^{2}\right)^{-1} \boldsymbol{C}_{0}^{\infty}(\Omega)$. 
In the last equality, we have used the fact that $I_{m} \varphi=\varphi m-$ a.e. This relation is not selfevident because in general $\varphi$ does not belong to $\boldsymbol{C}_{0}^{\infty}(\Omega)$. However, one can approximate it by $\boldsymbol{C}_{0}^{\infty}$-functions. More specifically, define $\varphi_{n}:=j_{n} \varphi$, where $j_{n} \in C_{0}^{\infty}(\Omega)$ such that $0 \leq j_{n}(x) \leq 1$ for all $x \in \Omega$ and $j_{n}(x)=1$ for $|x| \leq n$. Since $\varphi_{n} \rightarrow \varphi$ pointwise $d x-$ a.e. as $n \rightarrow \infty$ and $\left|\varphi_{n}\right| \leq|\varphi| \in \boldsymbol{W}_{0}^{2,1}(\Omega)$, it follows by the dominated convergence theorem that $\varphi_{n} \rightarrow \varphi$ in $\boldsymbol{W}_{0}^{2,1}(\Omega)$. From the definition of $I_{m}$, we get also $I_{m} \varphi_{n} \rightarrow I_{m} \varphi$ in $\boldsymbol{L}^{2}(m)$. Since $I_{m} \varphi_{n} \in \boldsymbol{C}_{0}^{\infty}(\Omega)$ by construction, we infer that $I_{m} \varphi_{n}=\varphi_{n}$ holds $m$ - a.e., and therefore $I_{m} \varphi=\varphi m$ - a.e. as well.

Since the set $\left(-\Delta_{D}^{\Omega}-k^{2}\right)^{-1} \boldsymbol{C}_{0}^{\infty}(\Omega)$ is dense in $\boldsymbol{L}^{1}(\Omega)$, it follows that

$$
\psi_{m}^{k}=R_{m, d x}^{k} \psi \quad d x-\text { a.e. }
$$

Now we can mimick the argument of [3] again: in the next step we consider a non-negative $\psi \in \boldsymbol{L}^{2}(m)$ and use a standard approximation argument choosing a sequence $\left\{\tilde{\psi}_{n}\right\}_{n=1}^{\infty} \subset \boldsymbol{L}^{1}(m) \cap \boldsymbol{L}^{2}(m)$ such that

$$
\lim _{n \rightarrow \infty} \tilde{\psi}_{n}=\psi \quad \text { and } \quad 0 \leq \tilde{\psi}_{1} \leq \tilde{\psi}_{2} \leq \cdots \leq \tilde{\psi}_{n} \quad m \text {-a.e. }
$$

Then

$$
\forall \varphi \in \boldsymbol{W}_{0}^{2,1}(\Omega): \quad\left\langle R_{m, d x}^{k} \tilde{\psi}_{n}, \varphi\right\rangle_{k}=\int_{\Omega} \overline{\tilde{\psi}_{n}(y)}\left(I_{m} \varphi\right)(y) d m(y)
$$

and by the dominated convergence theorem we get

$$
\forall \varphi \in \boldsymbol{W}_{0}^{2,1}(\Omega): \quad\left\langle R_{m, d x}^{k} \tilde{\psi}_{n}, \varphi\right\rangle_{k} \rightarrow \int_{\Omega} \bar{\psi}(y)\left(I_{m} \varphi\right)(y) d m(y)=\left\langle R_{m, d x}^{k} \psi, \varphi\right\rangle_{k}
$$

as $n \rightarrow \infty$, i.e., the sequence $\left\{R_{m, d x}^{k} \tilde{\psi}_{n}\right\}$ converges weakly to $R_{m, d x}^{k} \psi$ in the Hilbert space $\left(\boldsymbol{W}_{0}^{2,1}(\Omega),\langle\cdot, \cdot\rangle_{k}\right)$. By the diagonal trick [17, Sec. I.5] we may thus assume (selecting a subsequence if necessary) that

$$
R_{m, d x}^{k} \psi_{n} \rightarrow R_{m, d x}^{k} \psi \quad \text { as } \quad n \rightarrow \infty
$$

strongly in $\boldsymbol{W}_{0}^{2,1}(\Omega)$, where $\psi_{n}:=\frac{1}{n} \sum_{j=1}^{n} \tilde{\psi}_{j}$. Since $G_{0}(\cdot, \cdot ; k)$ is nonnegative and the sequence $\left\{\psi_{n}\right\}$ is nondecreasing again, the monotone convergence theorem implies

$$
\forall x \in \Omega: \quad \int_{\Omega} G_{0}(x, y ; k) \psi(y) d m(y)=\lim _{n \rightarrow \infty}\left(R_{m, d x}^{k} \psi_{n}\right)(x) \leq \infty .
$$


The relation (2.7) then gives

$$
\int_{\Omega} G_{0}(\cdot, y ; k) \psi(y) d m(y)=R_{m, d x}^{k} \psi \quad d x \text {-a.e. }
$$

Finally, by linearity the result extends to any $\psi \in \boldsymbol{L}^{2}(m)$.

It remains to establish the sought identity for an arbitrary $k \in \mathbb{C}_{\Omega, 0}^{+}$. To this aim, we employ the first resolvent relation which gives

$$
R_{m, d x}^{\tilde{k}}=R_{m, d x}^{k}+\left(\tilde{k}^{2}-k^{2}\right) R_{0}^{\tilde{k}} R_{m, d x}^{k}
$$

where we have denoted $R_{0}^{k}:=R_{d x, d x}^{k}$. Using repeatedly Lemma 2.1 we find

$$
\begin{aligned}
\mathcal{E}_{0}\left(R_{m, d x}^{\tilde{k}} \psi, \varphi\right)-\left(\tilde{k}^{2} R_{m, d x}^{\tilde{k}} \psi, \varphi\right) & \\
= & \mathcal{E}_{0}\left(R_{m, d x}^{k} \psi, \varphi\right)-\left(\tilde{k}^{2} R_{m, d x}^{k} \psi, \varphi\right) \\
& +\mathcal{E}_{0}\left(\left(\tilde{k}^{2}-k^{2}\right) R_{0}^{\tilde{k}} R_{m, d x}^{k} \psi, \varphi\right)-\left(\tilde{k}^{2}\left(\tilde{k}^{2}-k^{2}\right) R_{0}^{\tilde{k}} R_{m, d x}^{k} \psi, \varphi\right) \\
= & \mathcal{E}_{0}\left(R_{m, d x}^{k} \psi, \varphi\right)-\left(\tilde{k}^{2} R_{m, d x}^{k} \psi, \varphi\right)+\left(\tilde{k}^{2}\left(\tilde{k}^{2}-k^{2}\right) R_{0}^{\tilde{k}} R_{m, d x}^{k} \psi, \varphi\right) \\
& +\left(\left(\tilde{k}^{2}-k^{2}\right) R_{m, d x}^{k} \psi, \varphi\right)-\left(\tilde{k}^{2}\left(\tilde{k}^{2}-k^{2}\right) R_{0}^{\tilde{k}} R_{m, d x}^{k} \psi, \varphi\right) \\
& \\
= & \mathcal{E}_{0}\left(R_{m, d x}^{k} \psi, \varphi\right)-\left(k^{2} R_{m, d x}^{k} \psi, \varphi\right) .
\end{aligned}
$$

\subsection{The resolvent}

The above result allows us to write an explicit formula for the resolvent of $H_{\alpha m}$ and to derive some properties of it. We could just quote the results which we shall need in the following, but for the sake of completeness we sketch also the proofs which are essentially the same as in [3].

Proposition 2.3 Let $k \in \mathbb{C}_{\Omega, 0}^{+}$. Suppose that the operator $I+\alpha I_{m} R_{m, d x}^{k}$ is invertible on $\boldsymbol{L}^{2}(m)$ and the operator

$$
R^{k}:=R_{0}^{k}-R_{m, d x}^{k}\left(I+\alpha I_{m} R_{m, d x}^{k}\right)^{-1} \alpha I_{m} R_{0}^{k}
$$

is defined everywhere in $\boldsymbol{L}^{2}(\Omega)$. Then $k^{2} \in \rho\left(H_{\alpha m}\right)$ and $\left(H_{\alpha m}-k^{2}\right)^{-1}=R^{k}$. 
Proof: Take $\psi \in \boldsymbol{L}^{2}(\Omega)$ and $\varphi \in \boldsymbol{W}_{0}^{2,1}(\Omega) \equiv D\left(\mathcal{E}_{\alpha m}\right)$. By assumption, the operator $R^{k}$ is defined on $\boldsymbol{L}^{2}(\Omega)$. The free resolvent maps $\boldsymbol{L}^{2}(\Omega)$ into $\boldsymbol{W}_{0}^{2,1}(\Omega)$; the same is true for the second term in view of the assumed invertibility and Lemma 2.2. Thus $R^{k} \psi \in \boldsymbol{W}_{0}^{2,1}(\Omega)$, and by Lemma 2.1 we have to check that

$$
\mathcal{E}_{\alpha m}\left(R^{k} \psi, \varphi\right)-\left(k^{2} R^{k} \psi, \varphi\right)=(\psi, \varphi)
$$

holds for all $\psi, \varphi$ from the indicated sets. Dividing the left-hand-side into the "free" and "interaction" parts and denoting

$$
\chi:=\left(I+\alpha I_{m} R_{m, d x}^{k}\right)^{-1} \alpha I_{m} R_{0}^{k} \psi,
$$

we can rewrite it as

$$
\begin{gathered}
\mathcal{E}_{0}\left(R_{0}^{k} \psi, \varphi\right)-\left(k^{2} R_{0}^{k} \psi, \varphi\right)-\mathcal{E}_{0}\left(R_{m, d x}^{k} \chi, \varphi\right)+\left(k^{2} R_{m, d x}^{k} \chi, \varphi\right) \\
+\int_{\Omega} \alpha(x)\left(I_{m} \overline{R^{k} \psi}\right)(x)\left(I_{m} \varphi\right)(x) d m(x)
\end{gathered}
$$

The first two pair of terms equal $(\psi, \varphi)$ and $-\int_{\Omega} \bar{\chi}(x)\left(I_{m} \varphi\right)(x) d m(x)$ by Lemma 2.1 and Lemma 2.2, respectively. Since the relation should hold for all $\varphi \in \boldsymbol{W}_{0}^{2,1}(\Omega)$ we have thus to check that $\alpha I_{m} R^{k} \psi=\chi$ for any $\psi \in \boldsymbol{L}^{2}(\Omega)$, which follows by a simple algebraic manipulation,

$$
\begin{aligned}
& \alpha I_{m} R^{k} \psi=\alpha I_{m} R_{0}^{k} \psi-\alpha I_{m} R_{m, d x}^{k} \chi \\
& \quad=\left(I+\alpha I_{m} R_{m, d x}^{k} \psi\right) \underbrace{\left(I+\alpha I_{m} R_{m, d x}^{k} \psi\right)^{-1} \alpha I_{m} R_{0}^{k} \psi}_{\chi}-\alpha I_{m} R_{m, d x}^{k} \chi=\chi .
\end{aligned}
$$

As usual the invertibility assumption of the preceding proposition is satisfied for energies large enough negative.

Corollary 2.4 There is $\kappa_{0}>0$ such that $\left\|\alpha I_{m} R_{m, d x}^{i \kappa}\right\|<1$ for $\kappa \geq \kappa_{0}$.

Proof: In view of our basic assumption (2.1) and the boundedness of the operator $I_{m}$ we can choose $a<1$ and $0<b<\infty$ such that

$$
\forall \varphi \in \boldsymbol{W}_{0}^{2,1}(\Omega): \quad \int_{\Omega}\left|I_{m} \varphi(x)\right|^{2}\left(1+\alpha(x)^{2}\right) d m(x) \leq a\langle\varphi, \varphi\rangle_{i \kappa_{0}}
$$

where $\kappa_{0}:=\sqrt{\frac{b}{a}}$ and the inner product at the r.h.s. is defined in the proof of Lemma 2.2. We denote by $S_{\kappa}$ the unit sphere in $\left(\boldsymbol{W}_{0}^{2,1}(\Omega),\langle\cdot, \cdot\rangle_{i \kappa}\right)$. Given 
$\kappa \geq \kappa_{0}$ and $\psi \in \boldsymbol{L}^{2}(m)$, we deduce from the above inequality

$$
\begin{aligned}
& \int_{\Omega} \alpha(x)^{2}\left|\left(I_{m} R_{m, d x}^{i \kappa} \psi\right)(x)\right|^{2} d m(x) \leq a\left\langle R_{m, d x}^{i \kappa} \psi, R_{m, d x}^{i \kappa} \psi\right\rangle_{i \kappa_{0}} \\
& \leq a\left\langle R_{m, d x}^{i \kappa} \psi, R_{m, d x}^{i \kappa} \psi\right\rangle_{i \kappa}=a \sup _{\varphi \in S_{\kappa}}\left|\left\langle R_{m, d x}^{i \kappa} \psi, \varphi\right\rangle_{i \kappa}\right|^{2} .
\end{aligned}
$$

In view of Lemma 2.2, the last expression can be rewritten as

$$
\begin{aligned}
& a \sup _{\varphi \in S_{\kappa}}\left|\int_{\Omega} \bar{\psi}(y)\left(I_{m} \varphi\right)(y) d m(y)\right|^{2} \\
& \quad \leq a \int_{\Omega}|\psi(y)|^{2} d m(y) \sup _{\varphi \in S_{\kappa}} \underbrace{\int_{\Omega}\left|\left(I_{m} \varphi\right)(y)\right| d m(y)}_{\leq a\langle\varphi, \varphi\rangle_{i \kappa}} \leq a^{2} \int_{\Omega}|\psi(y)|^{2} d m(y)
\end{aligned}
$$

where we have used Schwarz inequality and the assumption (2.1) again. Consequently, $\left\|\alpha I_{m} R_{m, d x}^{i \kappa}\right\|<a$ holds for all $\kappa \geq \kappa_{0}$.

The resolvent expression of Proposition 2.3 represents a starting point for construction of the Birman-Schwinger theory. This will be done in the next section for our double-waveguide example. A part of the analysis is an expression for the number of eigenvalues of $H_{\alpha m}$ which can be derived in the present general context.

Corollary 2.5 $\operatorname{dim} \operatorname{Ker}\left(H_{\alpha m}-k^{2}\right)=\operatorname{dim} \operatorname{Ker}\left(I+\alpha I_{m} R_{m, d x}^{k}\right)$ holds for any $k \in \mathbb{C}_{\Omega, 0}^{+}$.

Proof: Suppose first that $\psi \in \boldsymbol{L}^{2}(m)$ satisfies $\psi+\alpha I_{m} R_{m, d x}^{k} \psi=0$. By Lemma 2.2 we have

$$
\begin{aligned}
& \mathcal{E}_{\alpha m}\left(R_{m, d x}^{k} \psi, \varphi\right)-\left(k^{2} R_{m, d x}^{k} \psi, \varphi\right) \\
& \quad=\int_{\Omega} \bar{\psi}(y)\left(I_{m} \varphi\right)(y) d m(y)+\int_{\Omega} \alpha(y)\left(I_{m} \overline{R_{m, d x}^{k} \psi}\right)(y)\left(I_{m} \varphi\right)(y) d m(y)=0
\end{aligned}
$$

for all $\varphi \in \boldsymbol{W}_{0}^{2,1}(\Omega) \equiv D\left(\mathcal{E}_{\alpha m}\right)$. By the second representation theorem, it follows that $R_{m, d x}^{k} \psi \in D\left(H_{\alpha m}\right)$ and $H_{\alpha m} R_{m, d x}^{k} \psi=k^{2} R_{m, d x}^{k} \psi$. Since $R_{m, d x}^{k}$ is injective by Lemma 2.2, we get

$$
\operatorname{dim} \operatorname{Ker}\left(I+\alpha I_{m} R_{m, d x}^{k}\right) \leq \operatorname{dim} \operatorname{Ker}\left(H_{\alpha m}-k^{2}\right) .
$$


On the other hand, let $\varphi \in D\left(H_{\alpha m}\right)$ with $H_{\alpha m} \varphi=k^{2} \varphi$. In view of the above argument, it is sufficient to show that $\varphi=R_{m, d x}^{k} \psi$ for some $\psi \in \boldsymbol{L}^{2}(m)$ such that $\psi+\alpha I_{m} R_{m, d x}^{k} \psi=0$. We put $\psi:=-\alpha I_{m} \varphi$; then by Lemma 2.2, we have

$$
\mathcal{E}_{0}\left(R_{m, d x}^{k} \psi, \chi\right)-\left(k^{2} R_{m, d x}^{k} \psi, \chi\right)=-\int_{\Omega} \alpha(x)\left(I_{m} \bar{\varphi}\right)(x)\left(I_{m} \chi\right)(x) d m(x)
$$

for all $\chi \in \boldsymbol{W}_{0}^{2,1}(\Omega)$. Using the second representation theorem again together with the assumption $H_{\alpha m} \varphi=k^{2} \varphi$ we get $\mathcal{E}_{\alpha m}(\varphi, \chi)-\left(k^{2} \varphi, \chi\right)=0$, so

$$
\begin{aligned}
\mathcal{E}_{0}(\varphi, \chi) & -\left(k^{2} \varphi, \chi\right) \\
& =\mathcal{E}_{\alpha m}(\varphi, \chi)-\left(k^{2} \varphi, \chi\right)-\int_{\Omega} \alpha(x)\left(I_{m} \bar{\varphi}\right)(x)\left(I_{m} \chi\right)(x) d m(x) \\
& =-\int_{\Omega} \alpha(x)\left(I_{m} \bar{\varphi}\right)(x)\left(I_{m} \chi\right)(x) d m(x)
\end{aligned}
$$

holds for any $\chi \in \boldsymbol{W}_{0}^{2,1}(\Omega)$. Comparing the two expressions, we arrive at the relation $\varphi=R_{m, d x}^{k} \psi$ which yields $\psi+\alpha I_{m} R_{m, d x}^{k} \psi=\psi+\alpha I_{m} \varphi=0$.

\section{$3 \quad$ BS analysis for the double waveguide}

The core of the classical Birman-Schwinger analysis is a resolvent expression containing at the l.h.s. only the free resolvent. If the Schrödinger operator in question involves a potential defined via a measure, the free resolvent has to be interpreted as an operator between different $\boldsymbol{L}^{2}$ spaces. Now we are going to derive such a formula for the system described in Sec. 1.2.

\subsection{The basic lemma}

The sought relation is an analogue of [3, Lemma 2.3] valid for $\Omega=\mathbb{R}^{d}$. The proof of this result employed the explicit form of the resolvent, thus the argument had to be modified again. We shall consider the operator $H_{\alpha m}$ of the previous section in the situation when $\Omega:=\mathbb{R} \times \mathcal{O}$ and $m$ is supported by the $x$-axis. Then we have:

Lemma 3.1 (i) $\forall k \in \mathbb{C}_{\Omega, 0}^{+}: I_{m} R_{m, d x}^{k}=R_{m, m}^{k}$ and $I_{m} R_{0}^{k}=R_{d x, m}^{k}$. (ii) $I+\alpha R_{m, m}^{i \kappa}$ has a bounded inverse on $\boldsymbol{L}^{2}(m)$ for all $\kappa>0$ large enough. (iii) Assume that $I+\alpha R_{m, m}^{k}$ is invertible for $k \in \mathbb{C}_{\Omega, 0}^{+}$and the operator

$$
R^{k}:=R_{0}^{k}-R_{m, d x}^{k}\left(I+\alpha R_{m, m}^{k}\right)^{-1} \alpha R_{d x, m}^{k}
$$


on $\boldsymbol{L}^{2}(m)$ is everywhere defined. Then $k^{2} \in \rho\left(H_{\alpha m}\right)$ and $\left(H_{\alpha m}-k^{2}\right)^{-1}=R^{k}$. (iv) $\forall k \in \mathbb{C}_{\Omega, 0}^{+}: \operatorname{dim} \operatorname{Ker}\left(H_{\alpha m}-k^{2}\right)=\operatorname{dim} \operatorname{Ker}\left(I+\alpha R_{m, m}^{k}\right)$.

ProOF: Since the assertions (ii)-(iv) are easy consequences of the first claim and the above corollaries, it is sufficient to check (i). The free Green's function for the strip $\Omega$ was written down in [7]. In particular, we have

$$
\begin{aligned}
G_{0}\left(\vec{x}, \vec{x}^{\prime} ; i \kappa\right) & =\frac{1}{D} \sum_{n=1}^{\infty} \frac{e^{-\kappa_{n}\left|x-x^{\prime}\right|}}{\kappa_{n}} \sin \frac{\pi n}{D}\left(y+d_{2}\right) \sin \frac{\pi n}{D}\left(y^{\prime}+d_{2}\right) \\
\kappa_{n} & :=\sqrt{\kappa^{2}+\left(\frac{\pi n}{D}\right)^{2}}
\end{aligned}
$$

for $\kappa>0$, where $\vec{x}:=(x, y)$. We know that $G_{0}\left(\vec{x}, \vec{x}^{\prime} ; i \kappa\right)>0$, it is smooth in each argument, exponentially decaying as $\left|x-x^{\prime}\right| \rightarrow \infty$ and has a logarithmic singularity as $\vec{x}^{\prime} \rightarrow \vec{x}$. As in Ref. [3], we need a smooth approximation to $G_{0}$. We employ the fact that

$$
G_{0}\left(\vec{x}, \vec{x}^{\prime} ; i \kappa\right)=-\frac{1}{2 \pi} \ln \left|\vec{x}-\vec{x}^{\prime}\right|+\Gamma\left(\vec{x}, \vec{x}^{\prime}\right),
$$

where $\Gamma$ is a $C^{\infty}$ function vanishing when $y, y^{\prime}$ assume the values $d_{1},-d_{2}$. We take

- a strictly increasing $\boldsymbol{C}^{\infty}$ function $\xi:(0, \infty) \rightarrow[1, \infty)$ such that $\xi(0)=1$ and $\xi(x)=x$ for $x \geq 2$,

- an increasing sequence $\left\{\eta_{n}\right\}_{n=1}^{\infty} \subset \boldsymbol{C}_{0}^{\infty}(\Omega)$ such that $\lim _{n \rightarrow \infty} \eta_{n}(\vec{x})=1$ for any fixed $\vec{x} \in \Omega$

and put

$$
G_{n}\left(\vec{x}, \vec{x}^{\prime} ; i \kappa\right):=\left[-\frac{1}{2 \pi} \ln \frac{\xi\left(n\left|\vec{x}-\vec{x}^{\prime}\right|\right)}{n}+\Gamma\left(\vec{x}, \vec{x}^{\prime}\right)\right] \eta_{n}(\vec{x})
$$

Clearly,

(i) $\quad G_{n}(\cdot, \vec{x} ; i \kappa) \in C_{0}^{\infty}(\Omega)$

(ii) $\quad \forall \vec{x}, \vec{x}^{\prime} \in \Omega: \quad G_{n}\left(\vec{x}, \vec{x}^{\prime} ; i \kappa\right) \leq G_{n+1}\left(\vec{x}, \vec{x}^{\prime} ; i \kappa\right)$

(iii) $\exists c_{1}>0 \forall \vec{x}, \vec{x}^{\prime} \in \Omega, \vec{x} \neq \vec{x}^{\prime}: \quad\left|\nabla_{x} G_{n}\left(\vec{x}, \vec{x}^{\prime} ; i \kappa\right)\right| \leq \frac{c_{1}}{\left|\vec{x}-\vec{x}^{\prime}\right|}$

(iv) $\exists c_{2}, c_{3}>0 \forall \vec{x}, \vec{x}^{\prime} \in \Omega,\left|\vec{x}-\vec{x}^{\prime}\right|$ large enough :

$$
\left|G_{n}\left(\vec{x}, \vec{x}^{\prime} ; i \kappa\right)\right|+\left|\nabla_{x} G_{n}\left(\vec{x}, \vec{x}^{\prime} ; i \kappa\right)\right| \leq c_{2} e^{-c_{3}\left|x-x^{\prime}\right|}
$$


We use the common notation $\mu$ for $m, d x$. Take an arbitrary $\psi \in \boldsymbol{L}^{2}(\mu)$ and $\varphi_{n}:=R_{\mu, d x}^{n, i \kappa} \psi$, i.e.

$$
\varphi_{n}=\int_{\Omega} G_{n}\left(\vec{x}, \vec{x}^{\prime} ; i \kappa\right) \psi(\vec{x}) d \mu\left(\vec{x}^{\prime}\right) .
$$

Each $\varphi_{n} \in \boldsymbol{W}_{0}^{2,1}(\Omega)$ by definition. Furthermore, by the construction of the regularized Green's function, we have also $\varphi_{n} \in \boldsymbol{C}_{0}^{\infty}(\Omega)$. Next we have to estimate the Sobolev norm of $\varphi_{n}$. In view of (iii) we have

$$
\begin{aligned}
\int_{\Omega} \mid & \left.\nabla_{x} \int_{\Omega} G_{n}\left(\vec{x}, \vec{x}^{\prime} ; i \kappa\right) \psi\left(x^{\prime}\right) d \mu\left(x^{\prime}\right)\right|^{2} d x \\
& =\int_{\Omega}\left|\int_{\Omega} \nabla_{x} G_{n}\left(\vec{x}, \vec{x}^{\prime} ; i \kappa\right) \psi\left(x^{\prime}\right) d \mu\left(x^{\prime}\right)\right|^{2} d x \\
& \leq \int_{\Omega}\left|\int_{\Omega} \frac{c_{1}}{\left|\vec{x}-\vec{x}^{\prime}\right|} \psi\left(x^{\prime}\right) d \mu\left(x^{\prime}\right)\right|^{2} d x \leq \int_{\mathbb{R}^{2}}\left|\int_{\mathbb{R}^{2}} \frac{c_{1}}{\left|\vec{x}-\vec{x}^{\prime}\right|} \psi\left(x^{\prime}\right) d \mu\left(x^{\prime}\right)\right|^{2} d x
\end{aligned}
$$

where $\psi$ in the last expression means the trivial extension from $\Omega$ to $\mathbb{R}^{2}$. The integral was shown to be finite in the proof of Lemma 2.3 in [3]. As for the non-derivative part we notice that $G_{0}$ has a bound as a consequence of the fact that $R_{\mu, d x}^{i \kappa} \psi \in \boldsymbol{W}_{0}^{2,1}(\Omega) \subset \boldsymbol{L}^{2}(\Omega)$.

Summing the above considerations, we have demonstrated that $\left\{\varphi_{n}\right\}$ is a bounded sequence in the local Sobolev space $\boldsymbol{W}_{0}^{2,1}(\Omega)$. Then we proceed as above: we construct $\chi_{n}:=\frac{1}{n} \sum_{j=1}^{n} \varphi_{j}$ and use the diagonal trick to show that (a subsequence of) $\left\{\chi_{n}\right\}$ converges strongly in $\boldsymbol{W}_{0}^{2,1}(\Omega)$. By the property (ii) and monotone convergence theorem

$$
\forall \vec{x} \in \Omega: \quad \lim _{n \rightarrow \infty} \chi_{n}(\vec{x})=\left(R_{\mu, d x}^{i \kappa} \psi\right)(\vec{x}),
$$

so $\chi_{n} \rightarrow R_{\mu, d x}^{i \kappa} \psi$ in $\boldsymbol{W}_{0}^{2,1}(\Omega)$ as $n \rightarrow \infty$. From the definition of $I_{m}$,

$$
I_{m} \chi_{n} \rightarrow I_{m} R_{\mu, d x}^{i \kappa} \psi \quad \text { in } \boldsymbol{L}^{2}(m)
$$

as $n \rightarrow \infty$. Since $I_{m} \chi_{n} \in \boldsymbol{C}_{0}^{\infty}(\Omega)$ by construction, we conclude that $I_{m} \chi_{n}=$ $\chi_{n}$ holds $m$-a.e., and therefore

$$
I_{m} R_{\mu, d x}^{i \kappa} \psi=R_{\mu, d x}^{i \kappa} \psi \quad m \text {-a.e. }
$$

This proves the desired relations for $k$ purely imaginary. The result extends to any $k \in \mathbb{C}_{\Omega, 0}^{+}$by means of the Hilbert identity as in the proof of Lemma 2.2. 


\subsection{The resolvent comparison formula}

From the point of view of our model the formula in Lemma 3.1(iii) still suffers from two defects. First of all, if we consider a semitransparent barrier whose coupling parameter is varied locally, it is natural to take $H_{\alpha m}$ with a constant but generally nonzero $\alpha$ as a comparison operator. Secondly, in analogy with the classical Birman-Schwinger theory it is useful to arrange the "potential" symmetrically with respect to the free resolvent in order to be able to use efficiently its decay properties.

Let $\alpha$ be as above a Borel measurable function $\mathbb{R} \rightarrow \mathbb{R}$ and $\alpha_{0} \in \mathbb{R}$; abusing the notation we shall employ the symbol $\alpha_{0}$ also for the constant function $\mathbb{R} \rightarrow \mathbb{R}, \alpha_{0}(x)=\alpha_{0}$. By the preceding result, we have

$$
R^{k}\left(\alpha_{0}\right)=R_{0}^{k}-R_{m, d x}^{k}\left(I+\alpha_{0} R_{m, m}^{k}\right)^{-1} \alpha_{0} R_{d x, m}^{k},
$$

so for any $k \in \mathbb{C}_{\Omega, 0}^{+}$with $k^{2} \in \rho\left(H_{\alpha_{0} m}\right) \cap \rho\left(H_{\alpha m}\right)$ :

$$
\begin{aligned}
R^{k}(\alpha)-R^{k}\left(\alpha_{0}\right)= & R_{m, d x}^{k}\left[\left(I+\alpha_{0} R_{m, m}^{k}\right)^{-1} \alpha_{0}-\left(I+\alpha R_{m, m}^{k}\right)^{-1} \alpha\right] R_{d x, m}^{k} \\
= & R_{m, d x}^{k}\left(I+\alpha R_{m, m}^{k}\right)^{-1}\left[\alpha_{0}\left(I+\alpha R_{m, m}^{k}\right)-\alpha\left(I+\alpha_{0} R_{m, m}^{k}\right)\right] \\
& \times\left(I+\alpha_{0} R_{m, m}^{k}\right)^{-1} R_{d x, m}^{k} \\
= & R_{m, d x}^{k}\left(I+\alpha R_{m, m}^{k}\right)^{-1}\left(\alpha_{0}-\alpha\right)\left(I+\alpha_{0} R_{m, m}^{k}\right)^{-1} R_{d x, m}^{k},(3.1)
\end{aligned}
$$

where in the second line we have used the fact that $\alpha_{0}$ is a number and thus commutes with $I+\alpha R_{m, m}^{k}$. Next we compute traces of $R^{k}\left(\alpha_{0}\right)$. By Lemma 3.1, we have

$$
\begin{aligned}
R_{d x, m}^{k}\left(\alpha_{0}\right) & =R_{d x, m}^{k}-R_{m, m}^{k}\left(I+\alpha_{0} R_{m, m}^{k}\right)^{-1} \alpha_{0} R_{d x, m}^{k} \\
& =\left(I+\alpha_{0} R_{m, m}^{k}\right)^{-1} R_{d x, m}^{k} .
\end{aligned}
$$

On the other hand, $\left(R_{\mu, \nu}^{k}\right)^{*}$ maps $\boldsymbol{L}^{2}(\nu)$ into $\boldsymbol{L}^{2}(\mu)$ and $\left(R_{\mu, \nu}^{k}\right)^{*}=R_{\nu, \mu}^{\bar{k}}$. In the same way as above, this yields

$$
R_{m, d x}^{k}\left(\alpha_{0}\right)=R_{m, d x}^{k}\left(I+\alpha_{0} R_{m, m}^{k}\right)^{-1} ;
$$

applying once more Lemma 3.1 we get also

$$
R_{m, m}^{k}\left(\alpha_{0}\right)=\left(I+\alpha_{0} R_{m, m}^{k}\right)^{-1} R_{m, m}^{k}=R_{m, m}^{k}\left(I+\alpha_{0} R_{m, m}^{k}\right)^{-1} .
$$

We employ these relations to proceed with the calculation of the resolvent difference (3.1): up to a sign change it equals

$$
R_{m, d x}^{k}\left(\alpha_{0}\right)\left(I+\alpha_{0} R_{m, m}^{k}\right)\left(I+\alpha R_{m, m}^{k}\right)^{-1}\left(\alpha-\alpha_{0}\right) R_{d x, m}^{k}\left(\alpha_{0}\right)
$$


and the central expression can be further rewritten as follows:

$$
\begin{aligned}
(I+ & \left.\alpha_{0} R_{m, m}^{k}\right)\left(I+\alpha R_{m, m}^{k}\right)^{-1}\left(\alpha-\alpha_{0}\right) \\
= & {\left[\left(I+\alpha_{0} R_{m, m}^{k}+\left(\alpha-\alpha_{0}\right) R_{m, m}^{k}\right)\left(I+\alpha_{0} R_{m, m}^{k}\right)^{-1}\right]^{-1}\left(\alpha-\alpha_{0}\right) } \\
= & {\left[I+\left(\alpha-\alpha_{0}\right) R_{m, m}^{k}\left(\alpha_{0}\right)\right]^{-1}\left(\alpha-\alpha_{0}\right) } \\
= & {\left[I+\left(\alpha-\alpha_{0}\right) R_{m, m}^{k}\left(\alpha_{0}\right)\right]^{-1}\left(\alpha-\alpha_{0}\right)^{\frac{1}{2}}\left[I+\left|\alpha-\alpha_{0}\right|^{\frac{1}{2}} R_{m, m}^{k}\left(\alpha_{0}\right)\left(\alpha-\alpha_{0}\right)^{\frac{1}{2}}\right] } \\
& \times\left[I+\left|\alpha-\alpha_{0}\right|^{\frac{1}{2}} R_{m, m}^{k}\left(\alpha_{0}\right)\left(\alpha-\alpha_{0}\right)^{\frac{1}{2}}\right]^{-1}\left|\alpha-\alpha_{0}\right|^{\frac{1}{2}} \\
= & {\left[I+\left(\alpha-\alpha_{0}\right) R_{m, m}^{k}\left(\alpha_{0}\right)\right]^{-1}\left[I+\left(\alpha-\alpha_{0}\right) R_{m, m}^{k}\left(\alpha_{0}\right)\right]\left(\alpha-\alpha_{0}\right)^{\frac{1}{2}} } \\
& \times\left[I+\left|\alpha-\alpha_{0}\right|^{\frac{1}{2}} R_{m, m}^{k}\left(\alpha_{0}\right)\left(\alpha-\alpha_{0}\right)^{\frac{1}{2}}\right]^{-1}\left|\alpha-\alpha_{0}\right|^{\frac{1}{2}} \\
= & \left(\alpha-\alpha_{0}\right)^{\frac{1}{2}}\left[I+\left|\alpha-\alpha_{0}\right|^{\frac{1}{2}} R_{m, m}^{k}\left(\alpha_{0}\right)\left(\alpha-\alpha_{0}\right)^{\frac{1}{2}}\right]^{-1}\left|\alpha-\alpha_{0}\right|^{\frac{1}{2}} .
\end{aligned}
$$

We employ here the usual "square-root convention" of the Birman-Schwinger theory, $\left(\alpha-\alpha_{0}\right)^{\frac{1}{2}}:=\left|\alpha-\alpha_{0}\right|^{\frac{1}{2}} \operatorname{sgn}\left(\alpha-\alpha_{0}\right)$.

Notice finally that the obtained expression no longer contains $R_{0}^{k}$. Using once again the Hilbert-identity trick, we can extend its validity to the set $\mathbb{C}_{\Omega, \alpha_{0}}^{+}:=\left\{k: \operatorname{Im} k>0\right.$ or $\left.k^{2} \in\left[0, \inf \sigma\left(H_{\alpha_{0} m}\right)\right)\right\}$. Summing up the above discussion, we get

Theorem 3.2 Under the stated assumptions, the resolvent of $H_{\alpha m}$ can be expressed by means of that of the reference operator $H_{\alpha_{0} m}$ as

$$
\begin{aligned}
R^{k}(\alpha)= & R^{k}\left(\alpha_{0}\right)-R_{m, d x}^{k}\left(\alpha_{0}\right)\left(\alpha-\alpha_{0}\right)^{\frac{1}{2}} \\
& \times\left[I+\left|\alpha-\alpha_{0}\right|^{\frac{1}{2}} R_{m, m}^{k}\left(\alpha_{0}\right)\left(\alpha-\alpha_{0}\right)^{\frac{1}{2}}\right]^{-1}\left|\alpha-\alpha_{0}\right|^{\frac{1}{2}} R_{d x, m}^{k}\left(\alpha_{0}\right)
\end{aligned}
$$

for any $k \in \mathbb{C}_{\Omega, \alpha_{0}}^{+}$.

Hence the original problem is equivalent to spectral analysis of the integral operator

$$
K_{\alpha}^{k}:=\left|\alpha-\alpha_{0}\right|^{\frac{1}{2}} R_{m, m}^{k}\left(\alpha_{0}\right)\left(\alpha-\alpha_{0}\right)^{\frac{1}{2}} .
$$

To be more specific we restrict ourselves to the situation which we shall discuss below and adopt the following assumptions:

(a1) $\alpha(\cdot)-\alpha_{0} \in \boldsymbol{L}^{1+\varepsilon}(\mathbb{R}, d x)$ for some $\varepsilon>0$, 
(a2) $\alpha(\cdot)-\alpha_{0} \in \boldsymbol{L}^{1}(\mathbb{R},|x| d x)$;

notice that as a consequence of (a1), (a2), the function belongs also to $\boldsymbol{L}^{1}(\mathbb{R}, d x)$.

Corollary 3.3 Under the assumption (a1), (a2),

(i) $K_{\alpha}^{k}$ is Hilbert-Schmidt for any $k \in \mathbb{C}_{\Omega, \alpha_{0}}^{+}$,

(ii) $I+K_{\alpha}^{i \kappa}$ has a bounded inverse on $\boldsymbol{L}^{2}(m)$ for all $\kappa>0$ large enough,

(iii) $\forall k \in \mathbb{C}_{\Omega, \alpha_{0}}^{+}: \operatorname{dim} \operatorname{Ker}\left(H_{\alpha m}-k^{2}\right)=\operatorname{dim} \operatorname{Ker}\left(I+K_{\alpha}^{k}\right)$,

(iv) Birman-Schwinger principle holds,

$$
\forall k \in \mathbb{C}_{\Omega, \alpha_{0}}^{+}: k^{2} \in \sigma_{\text {disc }}\left(H_{\alpha}\right) \Longleftrightarrow-1 \in \sigma_{\text {disc }}\left(K_{\alpha}^{k}\right) .
$$

ProOF: The Hilbert-Schmidt property follows by an argument analogous to the estimate of $N_{0}^{1}$ in Proposition 4.1 below, with the difference that the summation includes the first transverse mode too and $\sqrt{\nu_{n}-\nu_{1}}$ is replaced by $\sqrt{\nu_{n}-k^{2}}$. To prove (ii) one employs the analogue of (4.4) to infer that $\left\|K_{\alpha}^{i \kappa}\right\|_{H S}^{2} \rightarrow 0$ as $\kappa \rightarrow \infty$.

To deal with the rest, notice first that it is sufficient to prove (iii), (iv) for functions $\alpha$ which are essentially bounded. Indeed, define $\alpha_{N}(x):=$ $\operatorname{sgn} \alpha(x) \min \{|\alpha(x)|, N\}$. By absolute continuity of the Lebesgue integral the values of the quadratic form (2.2) related to $\alpha_{N}$ converge to that of $\mathcal{E}_{\alpha m}$ as $N \rightarrow \infty$ so $H_{\alpha_{N} m} \rightarrow H_{\alpha m}$ in the strong resolvent sense by [12, Thm. VIII.3.6]. Hence the discrete spectrum of $H_{\alpha m}$ is approximated by that of $H_{\alpha_{N} m}$ [17, Sec. VIII.7] but the latter has a finite dimension bound uniformly w.r.t. $N$ as we shall show in Proposition 6.1 .

Suppose therefore that $\left\|\alpha-\alpha_{0}\right\|_{\infty}<\infty$. The operator $I+K_{\alpha}^{k}$ has by (i) a purely discrete spectrum, every non-unit eigenvalue being of a finite multiplicity. Consequently, if $K_{\alpha}^{k}$ has the eigenvalue -1 , the number $k^{2}$ belongs to the spectrum of $H_{\alpha m}$ with the same multiplicity. On the other hand, if there is no $\psi$ solving $K_{\alpha}^{k} \psi=-\psi$, then $\left(I+K_{\alpha}^{k}\right)^{-1}$ is bounded, and so is $R^{k}(\alpha)$, thus $k^{2} \in \rho\left(H_{\alpha m}\right)$.

Remarks 3.4 (i) It is clear from (3.3) that there are other expressions of the resolvent, e.g.,

$$
R^{k}(\alpha)=R^{k}\left(\alpha_{0}\right)-R_{m, d x}^{k}\left(\alpha_{0}\right)\left[I+\left(\alpha-\alpha_{0}\right) R_{m, m}^{k}\left(\alpha_{0}\right)\right]^{-1}\left(\alpha-\alpha_{0}\right) R_{d x, m}^{k}\left(\alpha_{0}\right) .
$$

The advantage of the fully symmetric form is that it allows an optimal use of the decay properties of $\alpha-\alpha_{0}$. For instance, in the weak-coupling analysis 
of the next section the relation (3.5) would force us to restrict ourselves to the compact-support case.

(ii) As usual the BS principle provides an information about the discrete spectrum. Whether eigenvalues embedded in the essential spectrum may exist in the present situation remains an interesting open problem.

\section{Weak coupling}

Now we shall apply the above general results to weak-coupling analysis of our model represented by the Hamiltonian $H_{\alpha}$ defined in Section 2.1 (from now on we will omit the subscript $m$ ) which is considered as a perturbation of an $H_{\alpha_{0}}$ with a constant $\alpha_{0}$. The spectral properties of the latter operator are found easily; the corresponding analysis was done in [8, Sec. 2.2] where the reader can find a detailed account.

We have seen that the function $\alpha-\alpha_{0}$ plays the role of an effective potential. To introduce a parameter controlling the perturbation, we replace it in this section by $\lambda\left(\alpha-\alpha_{0}\right)$ with a small $\lambda$. Without loss of generality, we may suppose that the parameter is positive.

We shall concentrate on the case when the "leaky part" is localized in the sense that $\alpha(x)-\alpha_{0}$ decays fast enough as $|x| \rightarrow \infty$. Using a variational argument we have shown in [8] that the discrete spectrum is then non-empty. For a small positive $\lambda$ there is a unique bound state; our aim here is to derive an asymptotic expansion of the corresponding eigenvalue. The method follows the standard argument for one-dimensional Schrödinger operators [5, 19] and its extension to waveguide systems [6, Thm. 4.2.]. We shall suppose in the following that the assumptions (a1) and (a2) are valid.

\subsection{Preliminaries}

In the following we denote the ground-state eigenvalue of the weakly coupled Hamiltonian as $k^{2}$ and look for the function $\lambda \mapsto k^{2}$. Since we are interested in the discrete spectrum, we consider the Green's function for $k^{2}<\nu_{1}\left(\alpha_{0}\right)$, the threshold of the essential spectrum given by the first transverse eigenvalue of the unperturbed system - cf. [8, Sec. 3].

$$
K_{\alpha}\left(x, x^{\prime} ; k\right)=\left|\alpha(x)-\alpha_{0}\right|^{\frac{1}{2}} \sum_{n=1}^{\infty} \frac{\left|\chi_{n}\left(0 ; \alpha_{0}\right)\right|^{2}}{2 \kappa_{n}} e^{-\kappa_{n}\left|x-x^{\prime}\right|}\left(\alpha\left(x^{\prime}\right)-\alpha_{0}\right)^{\frac{1}{2}},
$$


where $\kappa_{n}:=\sqrt{\nu_{n}\left(\alpha_{0}\right)-k^{2}}$ and $\left\{\chi_{n}\right\}$ is the family the corresponding transverse eigenfunctions of the unperturbed system. It is straightforward to check that $\lim _{\lambda \rightarrow 0} k^{2}=\nu_{1}$, i.e., $\kappa_{1} \rightarrow 0$ as $\lambda \rightarrow 0$.

The key idea of the following argument is that $K_{\alpha}^{k}$ is well behaved in the limit $k^{2} \rightarrow \nu_{1}$ except for a divergent rank-one part. The singularity is contained in the first term of the expansion (4.1) and can be singled out by taking (as in) $K_{\alpha}^{k}=Q_{\alpha}+P_{\alpha}=Q_{\alpha}+A_{\alpha}+N_{\alpha}$ in analogy with [5, 6], where

$$
\begin{aligned}
& Q_{\alpha}\left(x, x^{\prime}\right)=\left|\alpha(x)-\alpha_{0}\right|^{\frac{1}{2}} e^{-\kappa_{1}|x|} \frac{\left|\chi_{1}(0)\right|^{2}}{2 \kappa_{1}} e^{-\kappa_{1}\left|x^{\prime}\right|}\left(\alpha\left(x^{\prime}\right)-\alpha_{0}\right)^{\frac{1}{2}} \\
& A_{\alpha}\left(x, x^{\prime}\right)=\left|\alpha(x)-\alpha_{0}\right|^{\frac{1}{2}} \frac{\left|\chi_{1}(0)\right|^{2}}{\kappa_{1}} e^{-\kappa_{1}|x|>} \sinh \kappa_{1}|x|_{<}\left(\alpha\left(x^{\prime}\right)-\alpha_{0}\right)^{\frac{1}{2}} \\
& N_{\alpha}\left(x, x^{\prime}\right)=\left|\alpha(x)-\alpha_{0}\right|^{\frac{1}{2}} \sum_{n=2}^{\infty} \frac{\left|\chi_{n}(0)\right|^{2}}{2 \kappa_{n}} e^{-\kappa_{n}\left|x-x^{\prime}\right|}\left(\alpha\left(x^{\prime}\right)-\alpha_{0}\right)^{\frac{1}{2}} .
\end{aligned}
$$

We have introduced here $|x|_{<}:=\max \left\{0, \min \left\{|x|,\left|x^{\prime}\right|\right\} \operatorname{sgn}\left(x x^{\prime}\right)\right\}$ and $|x|_{>}:=$ $\max \left\{|x|,\left|x^{\prime}\right|\right\}$; for the sake of brevity we drop $\alpha_{0}$ from the argument of $\chi_{n}$. Defining

$$
\begin{aligned}
A_{0}\left(x, x^{\prime}\right) & :=\left|\alpha(x)-\alpha_{0}\right|^{\frac{1}{2}}\left|\chi_{1}(0)\right|^{2}|x|_{<}\left(\alpha\left(x^{\prime}\right)-\alpha_{0}\right)^{\frac{1}{2}} \\
N_{0}^{\beta}\left(x, x^{\prime}\right) & :=\left|\alpha(x)-\alpha_{0}\right|^{\frac{1}{2}} \sum_{n=2}^{\infty} \frac{\left|\chi_{n}(0)\right|^{2}}{2} \frac{e^{-\beta \sqrt{\nu_{n}-\nu_{1}}\left|x-x^{\prime}\right|}}{\beta \sqrt{\nu_{n}-\nu_{1}}}\left(\alpha\left(x^{\prime}\right)-\alpha_{0}\right)^{\frac{1}{2}}
\end{aligned}
$$

with $\beta>0$, we get

Proposition 4.1 Let the assumptions (a1), (a2) be valid, then

$$
\lim _{\kappa_{1} \rightarrow 0}\left\|A_{\alpha}-A_{0}\right\|_{H S}=0 \quad \text { and } \quad \lim _{\kappa_{1} \rightarrow 0}\left\|N_{\alpha}-N_{0}^{1}\right\|_{H S}=0 .
$$

Proof: $\quad A_{0}$ is Hilbert-Schmidt since

$$
\begin{aligned}
\left\|A_{0}\right\|_{H S}^{2} & =\int_{\mathbb{R}^{2}}\left|A_{0}\left(x, x^{\prime}\right)\right|^{2} d x d x^{\prime} \\
& =\left|\chi_{1}(0)\right|^{4} \int_{\mathbb{R}^{2}}\left|\alpha(x)-\alpha_{0}\right||x|_{<}^{2}\left|\alpha\left(x^{\prime}\right)-\alpha_{0}\right| d x d x^{\prime} \\
& \leq\left|\chi_{1}(0)\right|^{4}\left(\int_{\mathbb{R}}|x|\left|\alpha(x)-\alpha_{0}\right| d x\right)^{2}<\infty
\end{aligned}
$$


by assumption. We have $\lim _{\kappa_{1} \rightarrow 0} A_{\alpha}=A_{0}$ and $\left|A_{\alpha}\left(x, x^{\prime} ; \kappa_{1}\right)\right| \leq\left|A_{0}\left(x, x^{\prime}\right)\right|$. This allows us to use the dominated convergence theorem which yields immediately the first claim.

$N_{0}^{1}$ has a logarithmic singularity as $x^{\prime} \rightarrow x$. Nevertheless, its HilbertSchmidt norm is finite because

$$
\begin{aligned}
\left\|N_{0}^{1}\right\|_{H S}^{2}= & \sum_{m, n=2}^{\infty} \frac{\left|\chi_{n}(0) \chi_{m}(0)\right|^{2}}{4 \sqrt{\nu_{n}-\nu_{1}} \sqrt{\nu_{m}-\nu_{1}}} \\
& \times \int_{\mathbb{R}^{2}}\left|\alpha(x)-\alpha_{0}\right| e^{-\left(\sqrt{\nu_{n}-\nu_{1}}+\sqrt{\nu_{m}-\nu_{1}}\right)\left|x-x^{\prime}\right|}\left|\alpha\left(x^{\prime}\right)-\alpha_{0}\right| d x d x^{\prime},
\end{aligned}
$$

where the monotone convergence theorem justifies the interchange of summation and integration, and by Hölder inequality the integral can be estimated by

$$
\begin{aligned}
&\left\|\alpha-\alpha_{0}\right\|_{1+\varepsilon} \int_{\mathbb{R}} d x\left|\alpha(x)-\alpha_{0}\right| \\
& \times\left\{e^{-\left(\sqrt{\nu_{n}-\nu_{1}}+\sqrt{\nu_{m}-\nu_{1}}\right) x}\left(\int_{-\infty}^{x} e^{\left(\varepsilon^{\prime}\right)^{-1}\left(\sqrt{\nu_{n}-\nu_{1}}+\sqrt{\nu_{m}-\nu_{1}}\right) x^{\prime}} d x^{\prime}\right)^{\varepsilon^{\prime}}\right. \\
&\left.+e^{\left(\sqrt{\nu_{n}-\nu_{1}}+\sqrt{\nu_{m}-\nu_{1}}\right) x}\left(\int_{x}^{\infty} e^{-\left(\varepsilon^{\prime}\right)^{-1}\left(\sqrt{\nu_{n}-\nu_{1}}+\sqrt{\nu_{m}-\nu_{1}}\right) x^{\prime}} d x^{\prime}\right)^{\varepsilon^{\prime}}\right\} \\
&=2\left(\varepsilon^{\prime}\right)^{\varepsilon^{\prime}}\left\|\alpha-\alpha_{0}\right\|_{1+\varepsilon}\left\|\alpha-\alpha_{0}\right\|_{1}\left(\sqrt{\nu_{n}-\nu_{1}}+\sqrt{\nu_{m}-\nu_{1}}\right)-\varepsilon^{\prime}
\end{aligned}
$$

where $\varepsilon^{\prime}$ is an abbreviation for $\frac{\varepsilon}{1+\varepsilon}>0$. The sequence $\left\{\chi_{n}(0)\right\}$ is uniformly bounded. To see this one has to employ the explicit expression of the transverse eigenfunctions given by the relations (2.7) and (2.8) of [8]. It implies the estimate $\chi_{n}(0)^{2} \leq 2 h_{1}(u) h_{2}(u)$, where $u:=\sqrt{\nu_{n}}$ and

$$
h_{j}(u):=\frac{\sqrt{u}\left|\sin d_{j} u\right|}{\sqrt{2 d_{j} u-\sin 2 d_{j} u}}
$$

for $j=1,2$. These functions are bounded in $(0, \infty)$ because they are continuous inside the interval and the limits

$$
\lim _{u \rightarrow 0+} h_{j}(u)=\left(2 d_{j}\right)^{-\frac{1}{2}} \quad \limsup _{u \rightarrow \infty} h_{j}(u)=\left(3 /\left(2 d_{j}\right)\right)^{-\frac{1}{2}}
$$

are finite. Consequently, it is sufficient to check the convergence of

$$
\sum_{m, n=2}^{\infty} \frac{1}{\sqrt{\nu_{n}-\nu_{1}} \sqrt{\nu_{m}-\nu_{1}}\left(\sqrt{\nu_{n}-\nu_{1}}+\sqrt{\nu_{m}-\nu_{1}}\right) \varepsilon^{\prime}}
$$


however, $\nu_{n}^{-1 / 2}=o\left(n^{-1}\right)$ as $n \rightarrow \infty-c f$. [8, Lemma 2.2]. The assumptions of the dominated convergence theorem are fulfilled again because $\lim _{\kappa_{1} \rightarrow 0} N_{\alpha}=$ $N_{0}^{1}$ and $\left|N_{\alpha}\left(x, x^{\prime} ; \kappa_{1}\right)\right| \leq\left|N_{0}^{1}\left(x, x^{\prime}\right)\right|$.

We will also need the boundedness of $A_{\alpha}$ and $N_{\alpha}$ for complex $z:=\kappa_{1}$. It follows from the above results, since it is easy to prove

Lemma 4.2 (i) $\forall z \in \mathbb{C}, \operatorname{Re} z \geq 0,|z|<\frac{\pi}{2}:\left|A_{\alpha}(z)\right| \leq \sqrt{2}\left|A_{0}\right|$ (ii) $\exists C, \beta>0 \quad \forall z \in \mathbb{C}, \operatorname{Re} z \geq 0,|z|<\sqrt{\nu_{n}-\nu_{1}}:\left|N_{\alpha}(z)\right| \leq C\left|N_{0}^{\beta}\right|$.

\subsection{Existence of the ground state}

Now we want to prove the following basic result:

Theorem 4.3 Assume that the hypotheses (a1), (a2) are valid. Then $H_{\alpha}$ has at most one simple eigenvalue $E(\lambda)<\nu_{1}$ for small enough $\lambda$, and this happens if and only if the equation

$$
\kappa_{1}=-\frac{\lambda}{2}\left|\chi_{1}(0)\right|^{2}\left(e^{-\kappa_{1}|\cdot|}\left(\alpha-\alpha_{0}\right)^{\frac{1}{2}},\left(I+\lambda P_{\alpha}\right)^{-1} e^{-\kappa_{1}|\cdot|}\left|\alpha-\alpha_{0}\right|^{\frac{1}{2}}\right)
$$

for $\kappa_{1}:=\sqrt{\nu_{1}-E}$ has a positive solution.

Proof: It is clear from the proof of Proposition 4.1 that $\left\|P_{\alpha}\right\| \leq\left\|A_{0}\right\|_{H S}+$ $\left\|N_{0}\right\|_{H S}<\infty$, thus $\left\|\lambda P_{\alpha}\right\|<1$ holds for sufficiently small $\lambda$. Then $I+\lambda P_{\alpha}$ is invertible and we may write

$$
\left(I+\lambda K_{\alpha}^{k}\right)^{-1}=\left[I+\left(I+\lambda P_{\alpha}\right)^{-1} \lambda Q_{\alpha}\right]^{-1}\left(I+\lambda P_{\alpha}\right)^{-1} .
$$

It follows that $\lambda K_{\alpha}$ has eigenvalue -1 if and only if the same is true for $\left(I+\lambda P_{\alpha}\right)^{-1} \lambda Q_{\alpha}$. Since $Q_{\alpha}$ is a rank-one operator by (4.2), we can express it as $\left(I+\lambda P_{\alpha}\right)^{-1} \lambda Q_{\alpha}=(\psi, \cdot) \varphi$ with $\psi:=\lambda \frac{\left|\chi_{1}(0)\right|^{2}}{2 \kappa_{1}} e^{-\kappa_{1}|\cdot|}\left(\alpha(\cdot)-\alpha_{0}\right)^{\frac{1}{2}}$ and $\varphi:=\left(I+\lambda P_{\alpha}\right)^{-1} e^{-\kappa_{1}|\cdot|}\left|\alpha(\cdot)-\alpha_{0}\right|^{\frac{1}{2}}$; it has just one eigenvalue, namely $(\psi, \varphi)$. Putting it equal to -1 we get the condition (4.5).

This proves the theorem except for the assertion that (4.5) has at most one positive solution for $\lambda$ small and fixed. Since there is a one-to-one correspondence between eigenvalues of $H_{\alpha}$ and solutions of (4.5) and the number of eigenvalue cannot decrease after the replacement $\alpha-\alpha_{0} \mapsto-\left|\alpha-\alpha_{0}\right|$, we 
need only show that (4.5) has at most one solution when $\alpha-\alpha_{0} \leq 0$. In this case, (4.5) is equivalent to $z=G(z, \lambda)$ where

$$
G(z, \lambda):=\frac{\lambda}{2}\left|\chi_{1}(0)\right|^{2}\left(e^{-z|\cdot|}\left|\alpha-\alpha_{0}\right|^{\frac{1}{2}},\left(I+\lambda P_{\alpha}\right)^{-1} e^{-z|\cdot|}\left|\alpha-\alpha_{0}\right|^{\frac{1}{2}}\right) .
$$

To complete the proof, we need several lemmas. The symbols $C, C_{j}$ in the following are unspecified constants.

Lemma 4.4 If $\alpha-\alpha_{0} \not \equiv 0$, then $|z|^{-1} \leq C_{1} \lambda^{-1}$ holds for $\lambda$ small.

Proof: From (4.6) we see that any solution of $z=G(z, \lambda)$ for $\lambda$ small must obey $z=\frac{\lambda}{2}\left|\chi_{1}(0)\right|^{2} \int_{\mathbb{R}}\left|\alpha(x)-\alpha_{0}\right| d x+\mathcal{O}\left(\lambda^{2}\right)$, which yields the assertion provided $\alpha-\alpha_{0}$ is not identically zero.

Lemma 4.5 For sufficiently small $z, \quad\left\|\frac{\partial P_{\alpha}}{\partial z}\right\|<C_{2}|z|^{-1}$.

Proof: Let us choose a circular contour, $\varphi: s=z\left(1+e^{i t}\right), t \in[0,2 \pi)$. The operator-valued fuction $P_{\alpha}(\cdot)$ is real-analytic in the region $\operatorname{Re} z>0$ and has a bounded limit as $z \rightarrow 0+$. Hence Cauchy integral formula together with Lemma 4.2 gives

$$
\left|\frac{\partial P_{\alpha}}{\partial z}\right|=\left|\frac{1}{2 \pi i} \int_{0}^{2 \pi} \frac{P_{\alpha}(s) i z e^{i t} d t}{z^{2} e^{2 i t}}\right| \leq \frac{\sqrt{2}\left|A_{0}\right|+C\left|N_{0}^{\beta}\right|}{|z|},
$$

but $A_{0}, N_{0}^{\beta}$ have finite HS norms.

Lemma 4.6 For any $z_{0} \in \mathbb{R}$ there is $C_{3}>0$ such that for all $z \in\left[0, z_{0}\right]$ we have

$$
\left|\left(e^{-z|\cdot|}\left|\alpha-\alpha_{0}\right|^{\frac{1}{2}},\left[2|\cdot|+\frac{\partial P_{\alpha}}{\partial z}\right] e^{-z|\cdot|}\left|\alpha-\alpha_{0}\right|^{\frac{1}{2}}\right)\right| \leq C_{3}
$$

Proof: An explicit calculation shows that the partial derivative is finite and remains bounded as $z \rightarrow 0+$. The other contribution to the scalar product is finite because of the assumption (a2).

\section{Lemma 4.7}

$$
\exists C_{4}>0: \quad\left|\left(e^{-z|\cdot|}\left|\alpha-\alpha_{0}\right|^{\frac{1}{2}},\left[|\cdot| P_{\alpha}+P_{\alpha}|\cdot|\right] e^{-z|\cdot|}\left|\alpha-\alpha_{0}\right|^{\frac{1}{2}}\right)\right| \leq \frac{C_{4}}{|z|}
$$


ProOF: Since $x e^{-x} \leq e^{-1}$ for any $x>0$, using Schwarz inequality we infer that the expression is bounded by $e^{-1}|z|^{-1}\left\|\alpha-\alpha_{0}\right\|_{1}\left\|P_{\alpha}\right\|_{H S}$.

Now we are able to complete the proof. Using the elementary inequality $\left(I+\lambda P_{\alpha}\right)^{-1} \leq C_{5}\left(1-\lambda P_{\alpha}\right)$ valid for small $\lambda$ together with the preceding lemmas we get for $\left|z^{-1}\right| \leq C_{1} \lambda^{-1}$ and all sufficiently small $\lambda$ :

$$
\left|\frac{\partial G}{\partial z}\right| \leq C \lambda
$$

Suppose that $z_{1}, z_{2}$ are two solutions of the equation $z=G(z, \lambda)$. They have to fulfill

$$
\left|z_{1}-z_{2}\right|=\left|\int_{z_{1}}^{z_{2}} \frac{\partial G}{\partial z} d z\right| \leq\left|\int_{z_{1}}^{z_{2}}\right| \frac{\partial G}{\partial z}|d z| \leq C \lambda\left|z_{1}-z_{2}\right|,
$$

hence the uniqueness is ensured for $\lambda<C^{-1}$.

\subsection{Weak-coupling expansion}

The results of the previous section make it possible to derive a necessary and sufficient condition for existence of a weakly coupled state, and also to write an expansion of the bound-state energy.

Theorem 4.8 Assume (a1), (a2) and $\alpha-\alpha_{0} \not \equiv 0$. Then the operator $H_{\alpha}$ has an eigenvalue $E(\lambda)<\nu_{1}$ for all sufficiently small $\lambda>0$ if and only if $\int_{\mathbb{R}}\left(\alpha(x)-\alpha_{0}\right) d x \leq 0$. In such a case, the eigenvalue is unique, simple, and obeys

$$
\begin{aligned}
& \sqrt{\nu_{1}-E(\lambda)}=-\frac{\lambda}{2}\left|\chi_{1}(0)\right|^{2} \int_{\mathbb{R}}\left(\alpha(x)-\alpha_{0}\right) d x \\
& \quad-\frac{\lambda^{2}}{4}\left\{\left|\chi_{1}(0)\right|^{4} \int_{\mathbb{R}^{2}}\left(\alpha(x)-\alpha_{0}\right)\left|x-x^{\prime}\right|\left(\alpha\left(x^{\prime}\right)-\alpha_{0}\right) d x d x^{\prime}\right. \\
& \left.\quad-\left|\chi_{1}(0)\right|^{2} \sum_{n=2}^{\infty}\left|\chi_{n}(0)\right|^{2} \int_{\mathbb{R}^{2}}\left(\alpha(x)-\alpha_{0}\right) \frac{e^{-\sqrt{\nu_{n}-\nu_{1}}\left|x-x^{\prime}\right|}}{\sqrt{\nu_{n}-\nu_{1}}}\left(\alpha\left(x^{\prime}\right)-\alpha_{0}\right) d x d x^{\prime}\right\} \\
& \quad+\mathcal{O}\left(\lambda^{3}\right) .
\end{aligned}
$$

Proof: Using the implicit-function theorem we can check that (4.5) has a unique solution for small $\lambda$, and that it is given by (4.7). It remains to 
prove that such a solution is strictly positive. This is clearly true for small enough $\lambda$ if $\int\left(\alpha(x)-\alpha_{0}\right) d x<0$. If the integral is zero, we have to check that the quadratic term is positive, which can be done by using the Fourier transformation in the same way as in [6, Thm. 4.2].

\section{$5 \quad$ Narrow-window coupling}

\subsection{Motivation: squeezing the "leaky" part}

If the effective potential is attractive, $\int\left(\alpha(x)-\alpha_{0}\right) d x<0$ the formula (4.7) can be rephrased as

$$
E(\lambda)=\nu_{1}\left(\alpha_{0}\right)-c \lambda^{2}+\mathcal{O}\left(\lambda^{3}\right), \quad c:=\frac{\left|\chi_{1}(0)\right|^{4}}{4}\left(\int_{\mathbb{R}}\left(\alpha(x)-\alpha_{0}\right) d x\right)^{2} .
$$

Hence the asymptotic behaviour is similar to that of $\llbracket$, Thm. 1.2.] where a straight Dirichlet strip with a small protrusion is considered.

However, the supremum norm is not the only mean by which the perturbation weakness can be controlled. To see that recall the example [9] of a double waveguide separated by a Dirichlet barrier with a window of a width $\ell$. It is very different from the situation considered above, since the Dirichlet condition corresponds formally to $\alpha_{0}=\infty$. Nevertheless, it has a weakly coupled state if the window is narrow, $\ell \ll d$, where $d:=\max \left\{d_{1}, d_{2}\right\}$. It was conjectured in 9 that

$$
E(\ell)=\left(\frac{\pi}{d}\right)^{2}-c(\nu) \ell^{4}+\mathcal{O}\left(\ell^{5}\right)
$$

where the parameter $\nu$ describes the waveguide asymmetry,

$$
\nu:=\frac{\min \left\{d_{1}, d_{2}\right\}}{\max \left\{d_{1}, d_{2}\right\}}
$$

In [10] the conjecture was supported by proving two-sided bounds by multiples of $\ell^{4}$ for the energy gap. Recently Popov [15 proved that the formula (5.2) is valid with

$$
c(\nu)=\left\{\begin{array}{lll}
\left(\frac{2 \pi^{3}}{d^{3}}\right)^{2} & \cdots & \nu=1 \\
\left(\frac{\pi^{3}}{d_{+}^{3}}\right)^{2} & \cdots & \nu<1
\end{array}\right.
$$


where $d_{+}:=\max \left\{d_{1}, d_{2}\right\}$. Recall that a similar quartic behaviour is known from waveguides with a critical local deformation [11] as well as for slightly bent or broken tubes [1, 6] where the leading term in the energy gap is proportional to the fourth power of the bending angle.

While our method does not allow us to include the case of a Dirichlet barrier, since such a boundary condition changes the form domain of the Hamiltonian, it is useful to investigate in our setting the situation when the weak-coupling limit consists of squeezing the "leaky" part while keeping $\left\|\alpha-\alpha_{0}\right\|_{\infty}$ fixed. We will achieve that by by introducing a longitudinal scaling of the coupling function,

$$
\alpha_{\sigma}(x):=\alpha\left(\frac{x}{\sigma}\right),
$$

with the scaling parameter $\sigma \in(0,1]$ and considering the limit $\sigma \rightarrow 0+$.

The argument proceeds in a similar way as above. The main tool is again Corrolary 3.3(iv) which is not affected by the scaling. However, as Remark 5.7(i) below shows, one cannot apply now the implicit-function theorem to derive an expansion for $\kappa_{1}$ analogous to (4.7). To avoid this difficulty, we employ the simpler decomposition (5.5) inspired by [19. It requires a stronger decay, namely

(a2') $\alpha(\cdot)-\alpha_{0} \in \boldsymbol{L}^{1}\left(\mathbb{R},|x|^{2} d x\right)$.

Let us show how the above results look like in the changed setting.

\subsection{Modified lemmas}

First note that $\lim _{\sigma \rightarrow 0+} k^{2}=\nu_{1}$, i.e., $\kappa_{1} \rightarrow 0$ as $\sigma \rightarrow 0+$. We put

$$
K_{\alpha_{\sigma}}^{k}=L_{\alpha_{\sigma}}+P_{\alpha_{\sigma}}=L_{\alpha_{\sigma}}+M_{\alpha_{\sigma}}+N_{\alpha_{\sigma}}
$$

where the kernels are given by

$$
\begin{aligned}
L_{\alpha_{\sigma}}\left(x, x^{\prime}\right) & =\left|\alpha_{\sigma}(x)-\alpha_{0}\right|^{\frac{1}{2}} \frac{\left|\chi_{1}(0)\right|^{2}}{2 \kappa_{1}}\left(\alpha_{\sigma}\left(x^{\prime}\right)-\alpha_{0}\right)^{\frac{1}{2}} \\
M_{\alpha_{\sigma}}\left(x, x^{\prime}\right) & =\left|\alpha_{\sigma}(x)-\alpha_{0}\right|^{\frac{1}{2}} \frac{\left|\chi_{1}(0)\right|^{2}}{2 \kappa_{1}}\left(e^{-\kappa_{1}\left|x-x^{\prime}\right|}-1\right)\left(\alpha_{\sigma}\left(x^{\prime}\right)-\alpha_{0}\right)^{\frac{1}{2}} \\
N_{\alpha_{\sigma}}\left(x, x^{\prime}\right) & =\left|\alpha_{\sigma}(x)-\alpha_{0}\right|^{\frac{1}{2}} \sum_{n=2}^{\infty} \frac{\left|\chi_{n}(0)\right|^{2}}{2 \kappa_{n}} e^{-\kappa_{n}\left|x-x^{\prime}\right|}\left(\alpha_{\sigma}\left(x^{\prime}\right)-\alpha_{0}\right)^{\frac{1}{2}}
\end{aligned}
$$


Next we define

$$
\begin{aligned}
& M_{0_{\sigma}}\left(x, x^{\prime}\right):=-\left|\alpha_{\sigma}(x)-\alpha_{0}\right|^{\frac{1}{2}} \frac{\left|\chi_{1}(0)\right|^{2}}{2}\left|x-x^{\prime}\right|\left(\alpha_{\sigma}\left(x^{\prime}\right)-\alpha_{0}\right)^{\frac{1}{2}} \\
& N_{0_{\sigma}}^{\beta}\left(x, x^{\prime}\right):=\left|\alpha_{\sigma}(x)-\alpha_{0}\right|^{\frac{1}{2}} \sum_{n=2}^{\infty} \frac{\left|\chi_{n}(0)\right|^{2}}{2} \frac{e^{-\beta \sqrt{\nu_{n}-\nu_{1}}\left|x-x^{\prime}\right|}}{\beta \sqrt{\nu_{n}-\nu_{1}}}\left(\alpha_{\sigma}\left(x^{\prime}\right)-\alpha_{0}\right)^{\frac{1}{2}} .
\end{aligned}
$$

In analogy with Proposition 4.1 we have

Proposition 5.1 Let the assumptions (a1), (a2') be valid, then

$$
\lim _{\kappa_{1} \rightarrow 0}\left\|M_{\alpha_{\sigma}}-M_{0_{\sigma}}\right\|_{H S}=0 \quad \text { and } \quad \lim _{\kappa_{1} \rightarrow 0}\left\|N_{\alpha_{\sigma}}-N_{0_{\sigma}}^{1}\right\|_{H S}=0 .
$$

ProOF: One has to check the first assertion, because the second one in Proposition 4.1 does not change. The operator $M_{0_{\sigma}}$ is Hilbert-Schmidt,

$$
\left\|M_{0}\right\|_{H S}^{2} \leq \sigma^{4} \frac{\left|\chi_{1}(0)\right|^{4}}{4} \int_{\mathbb{R}^{2}}\left|\alpha(x)-\alpha_{0}\right|\left(|x|^{2}+\left|x^{\prime}\right|^{2}\right)\left|\alpha\left(x^{\prime}\right)-\alpha_{0}\right| d x d x^{\prime}<\infty .
$$

Since $\lim _{\kappa_{1} \rightarrow 0} M_{\alpha_{\sigma}}=M_{0_{\sigma}}$ and $\left|M_{\alpha_{\sigma}}\left(x, x^{\prime} ; \kappa_{1}\right)\right| \leq\left|M_{0_{\sigma}}\left(x, x^{\prime}\right)\right|$, the result follows by means of the dominated convergence theorem.

It is easy to check that the norms of the operators $M_{\alpha_{\sigma}}, N_{\alpha_{\sigma}}$ can be made arbitrarily small by choosing $\sigma$ small enough, because

$$
\left\|M_{\alpha_{\sigma}}\right\|_{H S}^{2} \leq K_{1} \sigma^{4} \quad \text { and } \quad\left\|N_{\alpha_{\sigma}}\right\|_{H S}^{2} \leq K_{2} \sigma .
$$

Lemma 4.2 remains valid without any changes. Since its first claim is obtained by an algebraic manipulation, it holds for the operator $M_{\alpha_{\sigma}}$ as well. Finally, it is easy to see that Lemmas 4.44 .6 modify as follows:

Lemma 5.2 If $\alpha-\alpha_{0} \not \equiv 0$, then $|z|^{-1} \leq C_{1} \sigma^{-1}$ for $\sigma$ small.

Lemma 5.3 For sufficiently small z,

$$
\left\|\frac{\partial P_{\alpha_{\sigma}}}{\partial z}\right\|<C_{2} \frac{\sqrt{\sigma}}{|z|} .
$$


Lemma 5.4

$$
\forall z_{0} \in \mathbb{R} \quad \exists C_{3}>0 \quad \forall z \in\left[0, z_{0}\right]:\left|\left(\left|\alpha_{\sigma}-\alpha_{0}\right|^{\frac{1}{2}}, \frac{\partial P_{\alpha_{\sigma}}}{\partial z}\left|\alpha_{\sigma}-\alpha_{0}\right|^{\frac{1}{2}}\right)\right| \leq C_{3} \sigma^{2}
$$

Proof: Here one has only to show that $\partial M_{\alpha_{\sigma}} / \partial z$ remains bounded as $z \rightarrow 0+$ but this is clear from an elementary inequality, $1-e^{-x}-x e^{-x} \leq x^{2}$ for any $x \geq 0$. The rest of the argument follows the proof of Lemma 4.6 with the rescaled integration variables, $\left(x, x^{\prime}\right) \mapsto \sigma\left(x, x^{\prime}\right)$.

\subsection{The results for the scaled case}

With these preliminaries we can now formulate and prove a counterpart of Theorem 4.3.

Theorem 5.5 Let the assumptions (a1), (a2') be valid. Then $H_{\alpha_{\sigma}}$ has for $\sigma$ small enough at most one simple eigenvalue $E(\sigma)<\nu_{1}$, and this happens if and only if

$$
\sqrt{\nu_{1}-E} \equiv \kappa_{1}=-\frac{\left|\chi_{1}(0)\right|^{2}}{2}\left(\left(\alpha_{\sigma}-\alpha_{0}\right)^{\frac{1}{2}},\left(I+P_{\alpha_{\sigma}}\right)^{-1}\left|\alpha_{\sigma}-\alpha_{0}\right|^{\frac{1}{2}}\right)
$$

has a solution $\kappa_{1}>0$.

Proof: We mimick the proof of Theorem 4.3. Since $\left\|P_{\alpha_{\sigma}}\right\|<1$ holds for small enough $\sigma$, we may write

$$
\left(I+K_{\alpha_{\sigma}}^{k}\right)^{-1}=\left[I+\left(I+P_{\alpha_{\sigma}}\right)^{-1} L_{\alpha_{\sigma}}\right]^{-1}\left(I+P_{\alpha_{\sigma}}\right)^{-1} .
$$

The operator $\left(I+P_{\alpha_{\sigma}}\right)^{-1} L_{\alpha_{\sigma}}$ has just one eigenvalue $(\psi, \varphi)$ with

$$
\begin{aligned}
\psi & :=\frac{\left|\chi_{1}(0)\right|^{2}}{2 \kappa_{1}}\left(\alpha_{\sigma}(\cdot)-\alpha_{0}\right)^{\frac{1}{2}} \\
\varphi & :=\left(I+P_{\alpha_{\sigma}}\right)^{-1}\left|\alpha_{\sigma}(\cdot)-\alpha_{0}\right|^{\frac{1}{2}} .
\end{aligned}
$$

Putting it equal to -1 we get the implicit equation (5.10). As above, we can introduce

$$
G(z, \sigma):=\frac{\left|\chi_{1}(0)\right|^{2}}{2}\left(\left|\alpha_{\sigma}-\alpha_{0}\right|^{\frac{1}{2}},\left(I+P_{\alpha_{\sigma}}\right)^{-1}\left|\alpha_{\sigma}-\alpha_{0}\right|^{\frac{1}{2}}\right)
$$


and derive the estimate

$$
\left|\frac{\partial G}{\partial z}\right|<C \sigma^{2}
$$

which means that the uniqueness is now ensured for $\sigma<C^{-1 / 2}$.

The bound-state criterion and the asymptotic expansion with respect to the scaling parameter $\sigma$ look now as follows:

Theorem 5.6 Let $\alpha$ satisfy the assumptions (a1), (a2') and $\alpha-\alpha_{0} \not \equiv 0$. Then $H_{\alpha_{\sigma}}$ has an eigenvalue $E(\sigma)<\nu_{1}$ for all $\sigma$ small enough if and only if $\int_{\mathbb{R}}\left(\alpha(x)-\alpha_{0}\right) d x \leq 0$. If this condition holds, then the eigenvalue is unique, simple, and obeys

$$
\begin{aligned}
& \sqrt{\nu_{1}-E(\sigma)}=-\frac{\sigma}{2}\left|\chi_{1}(0)\right|^{2} \int_{\mathbb{R}}\left(\alpha(x)-\alpha_{0}\right) d x \\
& \quad+\frac{\sigma^{2}}{4}\left|\chi_{1}(0)\right|^{2} \sum_{n=2}^{\infty}\left|\chi_{n}(0)\right|^{2} \int_{\mathbb{R}^{2}}\left(\alpha(x)-\alpha_{0}\right) \frac{e^{-\sigma \sqrt{\nu_{n}-\nu_{1}}\left|x-x^{\prime}\right|}}{\sqrt{\nu_{n}-\nu_{1}}}\left(\alpha\left(x^{\prime}\right)-\alpha_{0}\right) d x d x^{\prime} \\
& +\mathcal{O}\left(\sigma^{3}\right) .
\end{aligned}
$$

Proof: Writing

$$
\left(I+P_{\alpha_{\sigma}}\right)^{-1}=I-P_{0_{\sigma}}-\left(P_{\alpha_{\sigma}}-P_{0_{\sigma}}\right)+P_{\alpha_{\sigma}}^{2}\left(I+P_{\alpha_{\sigma}}\right)^{-1},
$$

we see that (5.10) has a unique solution for $\sigma$ small which is given by (5.12). It is only important at that to notice that although $\sigma$ does enter the expansion (5.13) explicitly, it appears after inserting (5.13) into (5.10) because of the integration. This is also why we know that the last term in (5.13) does not contribute to the leading term in (5.12).) The rest of the proof concerning the strict positivity of such a solution proceeds in exactly the same way as in Theorem 4.8 .

Remarks 5.7 (i) We cannot expand the exponential in the quadratic term of (5.12) because the sum may not converge.

(ii) Notice that owing to (5.9), the expansion (5.12) does not contain the term arising from $M_{0_{\sigma}}$. This is a substantial difference from the analogous expansion (4.7).

(iii) In order to be able to compare the present case with the pierced Dirichlet barrier mentioned in the opening of this section, one should perform the limit 
$\alpha_{0} \rightarrow \infty$ assuming that $\alpha \equiv 0$ holds on a small compact. We observe that $\chi_{n}(0)$ decays like $\mathcal{O}\left(\alpha_{0}^{-1}\right)$ as $\alpha_{0} \rightarrow \infty$, so the first term in (5.12) vanishes after the limit. To get the behaviour (5.2) one would need to interchange the limit with the summation in the next term; it is not clear whether this can be done.

\section{A bound on the number of eigenvalues}

\subsection{A general SKN-type bound}

It is known that while a naive application of the Birman-Schwinger technique fails to yield an estimate on the bound state number in dimensions one and two, a simple trick invented independently by Seto 18, Klaus 13, and Newton [14] does the job. In this section we apply the idea to the measure-induced interaction in a strip to get an upper bound for the number of eigenvalues of our Hamiltonian below the essential spectrum $\sigma_{\text {ess }}\left(H_{\alpha}\right)=$ $\left[\nu_{1}\left(\alpha_{0}\right), \infty\right)$. The argument follows closely the considerations of [3, Sec. 3], hence we put emphasis again on the modifications. Hereafter, we assume (a1), (a2') because we shall employ the simpler decomposition (5.5) to single out the singularity in the kernel of of $K_{\alpha}^{k}$.

We denote by $\gamma$ the negative part of $\alpha-\alpha_{0}$, i.e., $\gamma:=\max \left\{0,-\left(\alpha-\alpha_{0}\right)\right\}$, and put

$$
\begin{aligned}
& \mu_{1}(\lambda):= \inf \left\{\mathcal{E}_{\left(\alpha_{0}-\lambda \gamma\right) m}(\psi, \psi) \mid \psi \in \boldsymbol{W}_{0}^{2,1}(\Omega),\|\psi\|=1\right\} \\
& \mu_{n}(\lambda):=\sup _{\varphi_{j} \in \boldsymbol{L}^{2}(\Omega)} \inf \left\{\mathcal{E}_{\left(\alpha_{0}-\lambda \gamma\right) m}(\psi, \psi) \mid \psi \in \boldsymbol{W}_{0}^{2,1}(\Omega),\|\psi\|=1\right. \\
&\left.\left(\psi, \varphi_{j}\right)=0, j=1, \ldots, n-1\right\}
\end{aligned}
$$

for any $\lambda \in[0,1)$ and all $n \in \mathbb{N} \backslash\{0\}$. We recall that the measure $\gamma m$ is finite by assumption and belongs to the generalized Kato class ( $c f$. Section 2.1).

In analogy with [3, Lemma 3.3.] we find that $\lambda \mapsto \mu_{n}(\lambda)$ is a nonincreasing continuous function on $[0,1)$ and $\mu_{n}(0)=\nu_{1}\left(\alpha_{0}\right)$ for all $n \in \mathbb{N}$. Mimicking further the second part of the proof of Proposition 4.1 one can show that $K_{\alpha_{0}-\lambda \gamma}^{i \kappa}$ is compact for $\kappa$ large enough, since it has a finite HilbertSchmidt norm. It is useful to introduce the standard family of Schatten 
norms,

$$
\|K\|_{p}:=\left(\sum_{j \in J} s_{j}(K)^{p}\right)^{\frac{1}{p}}
$$

for all $1 \leq p<\infty$, where $\left\{s_{j}(K)\right\}_{j \in J}$ is the family of eigenvalues of $|K|$; each eigenvalue is counted according to its multiplicity as an eigenvalue of $|K|$. We denote by $N_{E}$ the number of eigenvalues (counting multiplicity) of $H_{\alpha}$ which are smaller than $E$, and by $\# A$ the cardinality of the set $A$.

The crux of the BS method is the recognition that the number $N_{E}$ is equal to the number of eigenvalues of $K_{\alpha}^{\sqrt{E}}$ that are not less than 1 . It immediately follows from the form version of the minimax principle that $N_{E} \leq N_{E}^{-}$, the number of eigenvalues (counting multiplicity) of $H_{\alpha_{0}-\gamma}$ smaller than $E$. In analogy with [3, Thm. 3.3.] we therefore have

Proposition 6.1 $N_{E} \leq \#\left\{j \in J \mid s_{j}\left(K_{\alpha_{0}-\gamma}^{\sqrt{E}}\right) \geq 1\right\}$ holds for $E<\nu_{1}\left(\alpha_{0}\right)$. In particular, we have $N_{E} \leq\left\|K_{\alpha_{0}-\gamma}^{\sqrt{E}}\right\|_{p}^{p}$ for any $1 \leq p<\infty$.

This is the naive application mentioned above. It is not satisfactory in our situation, since the corresponding Green's function in $K_{\alpha}^{k}$ diverges for $k^{2} \rightarrow \nu_{1}\left(\alpha_{0}\right)-c f$. (4.1). The SKN-trick is based on the observation that this singularity does not depend effectively on the spectral parameter and corresponds therefore to just one bound state which can be taken into account separately.

Theorem 6.2 Suppose that $\|\gamma\|_{1} \neq 0$. Then the number $N_{\nu_{1}\left(\alpha_{0}\right)}$ of eigenvalues (counting multiplicity) below the threshold of the essential spectrum of $H_{\alpha}$ satisfies the bound

$$
\begin{aligned}
& N_{\nu_{1}\left(\alpha_{0}\right)}-1 \leq \\
& \quad \frac{\left|\chi_{1}(0)\right|^{4}}{4\|\gamma\|_{1}^{2}} \int_{\mathbb{R}^{4}}\left|x_{1}-x_{2}\right|\left(\left|x_{1}-x_{2}\right|\right. \\
& \left.\quad+\left|x_{3}-x_{4}\right|-\left|x_{1}-x_{3}\right|-\left|x_{2}-x_{4}\right|\right) \prod_{i=1}^{4} \gamma\left(x_{i}\right) d x_{i} \\
& +\frac{1}{4\|\gamma\|_{1}^{2}} \sum_{m, n=2}^{\infty} \frac{\left|\chi_{m}(0) \chi_{n}(0)\right|^{2}}{\sqrt{\nu_{m}-\nu_{1}} \sqrt{\nu_{n}-\nu_{1}}} \int_{\mathbb{R}^{4}} e^{-\sqrt{\nu_{m}-\nu_{1}}\left|x_{1}-x_{2}\right|}\left(e^{-\sqrt{\nu_{n}-\nu_{1}}\left|x_{1}-x_{2}\right|}\right.
\end{aligned}
$$




$$
\begin{gathered}
\left.+e^{-\sqrt{\nu_{n}-\nu_{1}}\left|x_{3}-x_{4}\right|}-e^{-\sqrt{\nu_{n}-\nu_{1}}\left|x_{1}-x_{3}\right|}-e^{-\sqrt{\nu_{n}-\nu_{1}}\left|x_{2}-x_{4}\right|}\right) \prod_{i=1}^{4} \gamma\left(x_{i}\right) d x_{i} \\
-\frac{\left|\chi_{1}(0)\right|^{2}}{2\|\gamma\|_{1}^{2}} \sum_{n=2}^{\infty} \frac{\left|\chi_{n}(0)\right|^{2}}{\sqrt{\nu_{n}-\nu_{1}}} \int_{\mathbb{R}^{4}}\left|x_{1}-x_{2}\right|\left(e^{-\sqrt{\nu_{n}-\nu_{1}}\left|x_{1}-x_{2}\right|}\right. \\
\left.+e^{-\sqrt{\nu_{n}-\nu_{1}}\left|x_{3}-x_{4}\right|}-e^{-\sqrt{\nu_{n}-\nu_{1}}\left|x_{1}-x_{3}\right|}-e^{-\sqrt{\nu_{n}-\nu_{1}}\left|x_{2}-x_{4}\right|}\right) \prod_{i=1}^{4} \gamma\left(x_{i}\right) d x_{i} .
\end{gathered}
$$

PROOF: It is an obvious modification of the proofs in 14. Borrowing the notation from this article and taking (5.5), (5.6) into account, we can write

$$
K_{\alpha_{0}-\gamma}=\xi(\varphi, \cdot) \varphi+P_{\alpha_{0}-\gamma}
$$

with $\xi=-\frac{\left|\chi_{1}(0)\right|^{2}}{2 \kappa_{1}}\|\gamma\|_{1}, \varphi=\gamma^{1 / 2} /\|\gamma\|_{1}^{-1 / 2}$, and $P_{\alpha_{0}-\gamma}=M_{\alpha_{0}-\gamma}+N_{\alpha_{0}-\gamma}$. We use the inequality obtained in [14, pp. 123] for $\xi \rightarrow \infty$,

$$
N_{\nu_{1}\left(\alpha_{0}\right)}^{-} \leq 1+\operatorname{tr} P_{0}^{2}-2\left(\varphi, P_{0}^{2} \varphi\right)+\left(\varphi, P_{0} \varphi\right)^{2},
$$

and substitute $P_{0}=M_{0}+N_{0}^{1}$, where $M_{0}, N_{0}^{1}$ are given by (5.7); this leads to the desired result.

\subsection{A "rectangular well" example}

To illustrate the above result let us apply it to the example analyzed in 8 , Sec. 4] in which $\alpha$ is a steplike function:

$$
\alpha(x):=\left\{\begin{array}{lll}
\alpha_{1} & \text { if } & |x|<a \\
\alpha_{0} & \text { if } & |x| \geq a
\end{array}\right.
$$

for some real $\alpha_{1}<\alpha_{0}$. Under the last condition the waveguide has a nontrivial discrete spectrum. Since $\gamma:=\alpha_{0}-\alpha_{1}$ is a constant on its support, we can evaluate the integrals of Theorem 6.2 obtaining

$$
\begin{aligned}
& N_{\nu_{1}\left(\alpha_{0}\right)} \leq 1+\frac{8}{45}\left|\chi_{1}(0)\right|^{4} \gamma^{2} a^{4} \\
& \quad+\frac{\gamma^{2} a^{4}}{2} \sum_{m, n=2}^{\infty} \frac{\left|\chi_{m}(0) \chi_{n}(0)\right|^{2}}{\tilde{\kappa}_{m} \tilde{\kappa}_{n}}\left\{\frac{2}{a\left(\tilde{\kappa}_{m}+\tilde{\kappa}_{n}\right)}+\frac{4}{a^{2} \tilde{\kappa}_{m} \tilde{\kappa}_{n}}-\frac{1-e^{-2 a\left(\tilde{\kappa}_{m}+\tilde{\kappa}_{n}\right)}}{a^{2}\left(\tilde{\kappa}_{m}+\tilde{\kappa}_{n}\right)^{2}}\right.
\end{aligned}
$$




$$
\begin{aligned}
& +\frac{\left(1-e^{-2 a \tilde{\kappa}_{m}}\right)\left(1-e^{-2 a \tilde{\kappa}_{n}}\right)}{a^{3} \tilde{\kappa}_{m} \tilde{\kappa}_{n}\left(\tilde{\kappa}_{m}+\tilde{\kappa}_{n}\right)}-\frac{4\left[\tilde{\kappa}_{m}\left(1-e^{-2 a \tilde{\kappa}_{n}}\right)+\tilde{\kappa}_{n}\left(1-e^{-2 a \tilde{\kappa}_{m}}\right)\right]}{a^{3}\left(\tilde{\kappa}_{m} \tilde{\kappa}_{n}\right)^{2}} \\
& \left.+\frac{2\left[\tilde{\kappa}_{m}^{3}\left(1-e^{-2 a \tilde{\kappa}_{n}}\right)-\tilde{\kappa}_{n}^{3}\left(1-e^{-2 a \tilde{\kappa}_{m}}\right)\right]}{a^{3}\left(\tilde{\kappa}_{m} \tilde{\kappa}_{n}\right)^{2}\left(\tilde{\kappa}_{m}^{2}-\tilde{\kappa}_{n}^{2}\right)}+\frac{2\left(1-e^{-2 a \tilde{\kappa}_{m}}\right)\left(1-e^{-2 a \tilde{\kappa}_{n}}\right)}{a^{4} \tilde{\kappa}_{m}^{2} \tilde{\kappa}_{n}^{2}}\right\} \\
& -2\left|\chi_{1}(0)\right|^{2} \gamma^{2} a^{3} \sum_{n=2}^{\infty} \frac{\left|\chi_{n}(0)\right|^{2}}{\tilde{\kappa}_{n}}\left(-\frac{2}{3 a \tilde{\kappa}_{n}}+\frac{2}{a^{2} \widetilde{\kappa}_{n}^{2}}-\frac{1-e^{-2 a \tilde{\kappa}_{n}}}{3 a^{2} \tilde{\kappa}_{n}^{2}}-\frac{2}{a^{3} \tilde{\kappa}_{n}^{3}}\right. \\
& \left.+\frac{1-e^{-2 a \tilde{\kappa}_{n}}}{a^{4} \tilde{\kappa}_{n}^{4}}\right)
\end{aligned}
$$

where $\tilde{\kappa}_{n}$ abbreviates $\sqrt{\nu_{n}-\nu_{1}}$.

To assess this bound, compare it with the one following from a simple bracketing argument and the minimax principle [8, Sec. 4.1.] which reads

$$
N_{\nu_{1}\left(\alpha_{0}\right)} \leq 1+\left[\frac{2 a}{\pi} \sqrt{\nu_{1}\left(\alpha_{0}\right)-\nu_{1}\left(\alpha_{1}\right)}\right],
$$

where [.] denotes the entire part. The r.h.s. is a "linearly increasing" step function with respect to the wi ndow halfwidth $a$. In distinction to (6.14), however, the bracketing argument yields in this example also a tight lower bound which differ just by one from (6.15). The bound (6.14) is not only more complicated, but it increases much faster with $a$; the comparison illustrates once more that while the Birman-Schwinger method is efficient for weak coupling, it may provide results far from optimal for strongly coupled systems.

Acknowledgments. We benefited from a discussion with W. Renger as well as from useful remarks of the referee. The work has been partially supported by the GA AS Grant 1048801. 


\section{References}

[1] Y. Avishai, D. Bessis, B. G. Giraud, and G. Mantica: "Quantum bound states in open geometries", Phys. Rev. B44 (1991), 8028-8034.

[2] S. Albeverio, F. Gesztesy, R. Høegh-Krohn, and H. Holden: Solvable Models in Quantum Mechanics, Springer, Heidelberg 1988.

[3] J. F. Brasche, P. Exner, Yu. A. Kuperin, and P. Šeba: "Schrödinger operators with sigular interactions", J. Math. Anal. Appl. 184 (1994), 112-139.

[4] W. Bulla, F. Gesztesy, W. Renger, and B. Simon: "Weakly coupled bound states in quantum waveguides", Proc. Amer. Math. Soc. 127 (1997), 1487-1495.

[5] R. Blackenbecler, M. L. Goldberger, and B. Simon: "The bound states of weakly coupled long-range one-dimensional quantum hamiltonians", Ann. Phys. 108 (1977), 69-78.

[6] P. Duclos and P. Exner: "Curvature-induced bound states in quantum waveguides in two and three dimensions", Rev. Math. Phys. 7 (1995), 73-102.

[7] P. Exner, R. Gawlista, P. Šeba, and M. Tater: "Point interactions in a strip", Ann. Phys. 252 (1996), 133-179.

[8] P. Exner and D. Krejčiř́ḱk: "Quantum waveguides with a lateral semitransparent barrier: Spectral and scattering properties", J. Phys. A32 (1999), 4475-4494.

[9] P. Exner, P. Šeba, M. Tater, and D. Vaněk: "Bound states and scattering in quantum waveguides coupled laterally through a boundary window", J. Math. Phys. 37 (1996), 4867-4887.

[10] P. Exner and S. A. Vugalter: "Asymptotic estimates for bound states in quantum waveguides coupled laterally through a narrow window", Ann. Inst. H. Poincaré 65 (1996), 109-123.

[11] P. Exner and S.A. Vugalter: "Bound states in a locally deformed waveguide: the critical case", Lett. Math. Phys. 39 (1997), 57-69.

[12] T. Kato: Perturbation Theory for Linear Operators, Springer, Berlin 1966.

[13] N. Klaus: "On the bound state of Schrödinger operators in one dimension", Ann. Phys. 108 (1977), 288-300. 
[14] R. G. Newton: "Bounds on the number of bound states for the Schrödinger equation in one and two dimensions", J. Operator Theory 10 (1983), 119-125.

[15] I. Yu. Popov: "Asymptotics of bound state for laterally coupled waveguides", Rep. Math. Phys. 43 (1999), 427-437.

[16] M.M. Rao: Measure Theory and Integration, John Wiley \& Sons, New York 1987.

[17] M. Reed and B. Simon: Methods of Modern Mathematical Physics, I. Functional Analysis, II. Fourier Analysis.Self-Adjointness, III. Scattering Theory, IV. Analysis of Operators, Academic Press, New York, 1972-1979.

[18] N. Seto: "Bargmann's inequalities in spaces of arbitrary dimension", Publ. Res. Inst. Math. Sci. 9 (1974), 429-461.

[19] B. Simon: "The bound state of weakly coupled Schrödinger operators in one and two dimensions", Ann. Phys. 97 (1976), 279-288.

[20] P. Stollmann and J. Voigt: "Perturbation of Dirichlet forms by measures", Potential Analysis 5 (1996), 109-138. 